\title{
CROSS-COMMODITY SPOT PRICE MODELING WITH STOCHASTIC VOLATILITY AND LEVERAGE FOR ENERGY MARKETS
}

\author{
F. E. BENTH, *** University of Oslo \\ L. VOS, ${ }^{*}$ University of Oslo and University of Agder
}

\begin{abstract}
Spot prices in energy markets exhibit special features, such as price spikes, mean reversion, stochastic volatility, inverse leverage effect, and dependencies between the commodities. In this paper a multivariate stochastic volatility model is introduced which captures these features. The second-order structure and stationarity of the model are analyzed in detail. A simulation method for Monte Carlo generation of price paths is introduced and a numerical example is presented.
\end{abstract}

Keywords: Energy market; Ornstein-Uhlenbeck process; stochastic volatility; leverage; subordinator

2010 Mathematics Subject Classification: Primary 60G10

Secondary 91G20; 15A04; 60G51; 60H30;

$91 \mathrm{G} 60$

\section{Introduction}

Energy markets worldwide have been liberalized over the last decades to create liquid trading arenas for power commodities like electricity, gas, and coal. The markets are continuously developing, and in recent years gradually becoming more and more connected. For instance, new cables and pipelines between the UK, Scandinavia, and continental Europe integrate the energy markets. The integration between markets calls for sophisticated multivariate pricing models for power. This includes cross-commodity models for gas and electricity, say, but also models for electricity traded in different but integrated markets. In this paper we propose and analyse a multivariate spot price model for power.

Power spot prices have several distinct characteristics. The market prices move with the season, with high prices in winter due to heating, or in summer due to air-conditioning cooling. Prices also naturally mean revert due to demand and supply forces. Typically, spot prices spike occasionally when there is an imbalance in supply and demand, since the demand curve is inelastic. Partly because of these spikes, the prices observed in gas and electricity markets are to a large extent leptokurtic. In fact, power prices may rise by several magnitudes compared to their expected mean levels within short time intervals. A discussion of the features of power spot prices can be found in [18] and [19]. There exist many models for spot price dynamics in power markets, and we refer the reader to [13] for an overview and analysis.

In energy markets there is evidence of a so-called inverse leverage effect. The volatility tends to increase with the level of power prices, since there is a negative relationship between

Received 22 November 2011; revision received 24 October 2012.

* Postal address: Centre of Mathematics for Applications, University of Oslo, PO Box 1053, Blindern, N-0316 Oslo, Norway.

** Email address: fredb@math.uio.no 
inventory and prices (see, for instance, [17]). Little available inventory means higher and more volatile prices. This is reflected in gas markets where storage facilities play an important role in price determination. There is also evidence for dependence between different commodities. For instance, it is unlikely that the price of gas and electricity in the UK market, say, will drive too far apart, since gas is the dominating fuel for power production. Likewise, since gas can be transported as liquid natural gas (LNG), different gas markets cannot have prices which become increasingly different.

In recent years there has been an interest in stochastic volatility models for commodities, and in particular energies. In Hikspoors and Jaimungal [20] we find an analysis of forward pricing in commodity markets in the presence of stochastic volatility. Several popular models are treated. More recently, Trolle and Schwartz [32] introduced the notion of unspanned volatility, and analyzed this in power markets. Their statistical analysis confirms the presence of stochastic volatility in commodity markets. Benth [8] applied the Barndorff-Nielsen and Shephard stochastic volatility model in commodity markets, and derived forward prices based on this. An empirical study on UK gas prices was performed.

In this paper we propose stochastic dynamics for cross-commodity spot price modeling, generalizing the univariate dynamics studied in [8]. The model is flexible enough to capture spikes, mean reversion, and stochastic volatility. Moreover, it includes the possibility to model inverse leverage. Our proposed dynamics can model co- and independent jump behavior (spikes) in cross-commodity markets.

The spot price dynamics we propose are based on Ornstein-Uhlenbeck processes driven by multivariate subordinators. The logarithmic price dynamics are defined by multifactor processes and seasonal functions to account for deterministic variability over a year. The stochastic volatility processes are multivariate as well, so that we can incorporate secondorder dependencies between commodities. The volatility model is adopted from the so-called Barndorff-Nielsen and Shephard model (BNS model for short), extended to a multivariate setting (see [4] and [7]). As for the multidimensional extension, the volatility is modeled with a matrix-valued Ornstein-Uhlenbeck process driven by a positive definite matrix-valued subordinator (see [2]). We prove that the spot price converges to a stationary dynamic, and compute the characteristic function of the limiting distribution. Several other probabilistic features of the model are presented and discussed, demonstrating its flexibility in modeling prices and its analytical tractability. From a more practical point of view, a method for simulating the prices is presented. We provide an empirical example where the algorithm is applied. Our approach is influenced by the work of Stelzer [31].

One of the main features of our spot price model is its explicit characteristics. We are able to derive reasonably analytic cumulant (logarithmic characteristic) functions for the spot model and its factors. These cumulants are important ingredients in many different applications of the model, as well as crucial for the study of the probabilistic properties of the price dynamics. From the cumulants we can show that the spot price converges to a stationary dynamic, and, moreover, characterize the limiting distribution. In this respect, our proposed model is a true multivariate non-Gaussian extension of the classical univariate Ornstein-Uhlenbeck process suggested by Schwartz [29] for commodity prices. The first- and second-order moments are analytically expressible in terms of the parameters of the model. From this we recover a rather flexible autocovariance structure of the price dynamics, being asymptotically a linear combination of exponentially decaying functions. One may apply this analytic autocovariance structure to calibrate data, as several studies show that energy prices have a decaying empirical autocovariance function which may be represented as a series of exponential functions. 
Frequently, one associates the different exponentials in the autocovariance function to different factors in the spot dynamics. This is indeed the case of our model, where, for example, spike factors enter the autocovariance explicitly. We also obtain the covariance structure for the factors in the multivariate spot price dynamics.

In a companion paper [11], we derive the implied forward and futures price dynamics from our spot price model. Forward and futures contracts are liquidly traded in most energy markets, like oil, gas, and power. Moreover, derivatives written on these contracts are traded in many markets. For example, the German power market EEX organizes standard European call and put options with forward and futures contracts as underlying. At the NYMEX market in New York, options written on the spread between refined oil futures of different qualities are traded (so-called crack spreads). In the markets, there also exist options of various kinds written on the spread between power and gas futures (spark spreads), or power and coal futures (dark spread). Without a reasonably explicit dynamic for the futures price, the analysis of such derivatives becomes very complicated. However, within our class of spot price models, we can derive expressions for the forward and futures price dynamics which allow for efficient pricing of derivatives based on Fourier methods. These methods make explicit use of the characteristic functions of the factors in the spot dynamics.

The cumulant functions of the spot dynamics can also be the basis for developing estimation techniques for the model in the Fourier (frequency) domain. For a rather general class of affine models, this has been thoroughly analyzed by Singleton [30]. Here, moment estimators are proposed based on the empirical cumulants, as well as different time-domain estimators based on Fourier inversion of the cumulants. In future studies it would be interesting to adapt these methods for the particular class of spot price dynamics proposed in our paper, and apply them to estimation on energy data.

Our assumptions on the parameters in the proposed spot price dynamics are rather strong. We model the logarithmic deseasonalized spot prices and the stochastic volatility as multivariate and matrix-valued Ornstein-Uhlenbeck processes. In such models, we model the mean reversion as well as the mixing between the marginals, opening up for a functional dependence between the factors, and thereby the marginal spot dynamics. However, as it turns out, this flexibility is rather narrow, as the involved matrices for some of the factors must commute and be invertible. This constraint on the parameters comes from the fact that we model both the logarithmic deseasonalized spot prices and the stochastic volatility as Ornstein-Uhlenbeck processes in multiple dimensions. The issue of invertibility is natural, as it corresponds to a similar assumption for the univariate model considered in [8]. There a Schwartz model with stochastic volatility of the Barndorff-Nielsen and Shephard class was analyzed based on the inequality of the speed of mean reversion of the volatility process and the logarithmic spot. As it turns out, the additional commutativity condition enters into play when we consider multidimensional models. Dispensing with these conditions leads to rather complicated expressions for the cumulants, say.

Our model explicitly accounts for spikes behavior and possible (inverse) leverage effects. As the commutativity condition does not enter into this part of the model, we may have a direct relationship between spikes in different markets, for example, co-spikes in gas and electricity, or co-spikes in two connected power markets.

Recently, Muhle-Karbe et al. [23] proposed and analyzed multivariate geometric Brownian motion dynamics with a stochastic volatility model of Barndorff-Nielsen and Shephard type. Our spot price dynamics share some similarities with their asset price process, in particular the stochastic volatility part. However, our model has some features significantly extending 
their dynamics. Firstly, we assume the logarithmic prices to be a multivariate OrnsteinUhlenbeck process. This turns out to complicate the calculation of the cumulant functions, as well as giving some additional hypothesis on the parameters. Secondly, our additional spike and (inverse leverage) components are of a different form. As our model is aimed at energy markets, it will naturally be different from the asset price model suggested in [23].

The paper is organized as follows. In Section 2 we introduce the spot model, thereafter the stationary distribution and the probabilistic properties of the various factors of the model are deduced in Section 3. In Section 4 we deal with the same properties of the spot price model. Section 5 gives an empirical example and a method to perform Monte Carlo simulation of the model. Finally, in Section 6 we conclude.

\subsection{Notation}

For the convenience of the reader, we have collected some frequently used notation. We adopt the notation used by Pirgorsch and Stelzer [24]. Throughout this paper, we write $\mathbb{R}_{+}$for the positive real numbers and we denote the set of real $n \times n$ matrices by $M_{n}(\mathbb{R})$. We denote the group of invertible matrices by $\mathrm{GL}_{n}(\mathbb{R})$, the linear subspace of symmetric matrices by $\mathbb{S}_{n}$, the positive definite cone of symmetric matrices by $\mathbb{S}_{n}^{+}$. We denote by $I_{n}$ the $n \times n$ identity matrix, by $J_{n}(v)$ the operator $\mathbb{R}^{n} \rightarrow M_{n}(\mathbb{R})$ which creates a diagonal matrix with the vector $v \in \mathbb{R}^{n}$ on the diagonal, by $\operatorname{diag}(A)$ the vector in $\mathbb{R}^{n}$ consisting of the diagonal of the matrix $A \in M_{n}(\mathbb{R})$, and by $\sigma(A)$ the spectrum (the set of all eigenvalues) of a matrix $A \in M_{n}(\mathbb{R})$. The tensor (Kronecker) product of two matrices $A, B$ is written as $A \otimes B$. We denote by vec the well-known vectorization operator that maps the $n \times n$ matrices to $\mathbb{R}^{n^{2}}$ by stacking the columns of the matrices below one another. Furthermore, $\operatorname{tr}(A)$ denotes the trace of the matrix $A \in M_{n}(\mathbb{R})$, which is the sum of the elements on the diagonal. The transpose of the matrix $A \in M_{n}(\mathbb{R})$ is denoted $A^{\top}$, while $A_{i j}$ is the element of $A$ in the $i$ th row and $j$ th column. The unit vector with a 1 in the $i$ th coordinate and 0 s elsewhere is denoted by $e_{i}$. For $A \in M_{n}(\mathbb{R})$, we denote the operator $\boldsymbol{A}$ associated with the matrix $A$ as $\boldsymbol{A}: X \mapsto A X+X A^{\top}$. This operator can be represented as $\operatorname{vec}^{-1} \circ\left(\left(A \otimes I_{n}\right)+\left(I_{n} \otimes A\right)\right) \circ$ vec. Its inverse is denoted by $\boldsymbol{A}^{-1}$, which exists whenever $I \otimes A+A \otimes I$ is invertible. In this case, we can represent $\boldsymbol{A}^{-1}$ by $\operatorname{vec}^{-1} \circ\left(\left(A \otimes I_{n}\right)+\left(I_{n} \otimes A\right)\right)^{-1} \circ$ vec. Note that $A \otimes I_{n}+I_{n} \otimes A$ is equal to the Kronecker sum of the matrix $A$ with itself.

\section{The cross-commodity spot price model}

Suppose that we are given a complete filtered probability space $(\Omega, \mathcal{F}, \mathbb{P})$ equipped with the filtration $\left\{\mathcal{F}_{t}\right\}_{t \geq 0}$ satisfying the usual conditions (see, e.g. [26]). Assume that $m, n \in \mathbb{N}$ with $0 \leq m<n$. Let $\left\{\widetilde{L}_{j}(t)\right\}_{t \in \mathbb{R}^{+}} \in \mathbb{S}_{d}^{+}, j=1, \ldots, n$, be $n$ independent matrix-valued subordinators, as introduced in [2]. Furthermore, let $L_{i}, i=1, \ldots, m$, be $\mathbb{R}^{d}$-valued subordinators. A multivariate subordinator is a Lévy process which is increasing in each of its coordinates; see [6]. For $i=1, \ldots, m$, the vector-valued subordinators $L_{i}$ are formed by taking the diagonal of the matrix-valued subordinators $\widetilde{L}_{i}(t)$. This implies that $L_{i}$ will jump whenever $\widetilde{L}_{i}$ does. If one of the off-diagonal elements jumps, the diagonal element also has to jump in order to keep the volatility process $\widetilde{L}_{i}$ in the positive definite cone $\mathbb{S}_{d}^{+}$. The subordinators are assumed to be driftless, and we choose to work with the versions which are right continuous with left limits (RCLL for short). Moreover, let $W$ be a standard $\mathbb{R}^{d}$-valued Brownian motion independent of the subordinators. 
We define the spot price dynamics of $d$ commodities as follows: let

$$
S(t)=\Lambda(t) \circ \exp \left(X(t)+\sum_{i=1}^{m} Y_{i}(t)\right)
$$

where $\Lambda:[0, T] \mapsto \mathbb{R}_{+}^{d}$ is a vector of measurable seasonality functions, 'o' denotes the Hadamard product (that is, coordinatewise multiplication), and

$$
\begin{aligned}
& \mathrm{d} X(t)=A X(t) \mathrm{d} t+\Sigma(t)^{1 / 2} \mathrm{~d} W(t), \\
& \mathrm{d} Y_{i}(t)=\left(\mu_{i}+B_{i} Y_{i}(t)\right) \mathrm{d} t+\eta_{i} \mathrm{~d} L_{i}(t)
\end{aligned}
$$

for $i=1, \ldots, m$. The matrices $A$ and $B_{i}$ are supposed to be elements of $\mathrm{GL}_{d}(\mathbb{R})$, while $\eta_{i}$ belongs to $M_{d}(\mathbb{R})$ and $\mu_{i}$ is a vector in $\mathbb{R}^{d}$. To ensure the existence of stationary solutions, we assume that the eigenvalues of the matrices $A$ and $B_{i}$ have negative real parts. In order to have the Itô integral in (2) well defined, we suppose that $\Sigma(t)$ is an $\mathcal{F}_{t}$-adapted, matrix-valued stochastic process such that

$$
\mathbb{P}\left(\int_{0}^{T} \operatorname{tr}(\Sigma(t)) \mathrm{d} t<\infty\right)=1 .
$$

Here, $T<\infty$ is some terminal time for our energy markets. The entries of $\eta_{i}$ can be negative. So, although $L_{i}$ is an $\mathbb{R}^{d}$-valued subordinator, there can be negative jumps in the spot price process.

The stochastic volatility process $\Sigma(t)$ is a superposition of the positive-definite matrix-valued Ornstein-Uhlenbeck process as introduced in [7], i.e.

$$
\Sigma(t)=\sum_{j=1}^{n} \omega_{j} Z_{j}(t),
$$

with

$$
\mathrm{d} Z_{j}(t)=\left(C_{j} Z_{j}(t)+Z_{j}(t) C_{j}^{\top}\right) \mathrm{d} t+\mathrm{d} \widetilde{L}_{j}(t),
$$

where the $\omega_{j}$ s are weights summing to 1 . Moreover, $\left\{C_{j}\right\}_{1 \leq j \leq n} \in \mathrm{GL}_{d}(\mathbb{R})$. To ensure that $Z_{j}$ has a limiting distribution (stationary distribution) when $t \rightarrow \infty$, we will assume that the eigenvalues of $C_{j}$ have negative real parts. This stochastic volatility model is a multivariate extension of the so-called BNS SV model introduced by Barndorff-Nielsen and Shephard [4] for general asset price processes. The commodity spot price dynamics with the BNS SV model as the stochastic volatility structure is a generalization to cross-commodity markets of the univariate spot model analyzed in [8].

Note that $Y_{i}$ and $\Sigma_{i}$ for $i=1, \ldots, m$ have related subordinators $L$ and $\widetilde{L}$ driving the noise. Thus, when the volatility component $\Sigma$ jumps, we observe simultaneously a change in the spot price. Hence, we can have an inverse leverage effect since increasing prices follow from increasing volatility, and vice versa (see [18] and [19] for a discussion on inverse leverage in power markets). We also have $n-m$ independent stochastic volatility components $Z_{j}$, $j=m+1, \ldots, n$, that do not directly influence the price process paths but have a second-order effect. The processes $Y_{i}$ can be interpreted as modeling the spikes, whereas $X$ models the normal variations in the market. The latter is also sometimes referred to as the base component of the price variations. 
By turning off the influence from the processes $Y_{i}$ (choose $m=0$ ), we obtain a multivariate extension of the Schwartz model with stochastic volatility and spot price dynamics

$$
S(t)=\Lambda(t) \circ \exp (X(t))
$$

where $X(t)$ is defined in (2). The Schwartz model with constant volatility is a mean-reversion process proposed by Schwartz [29] for the spot price dynamics in commodity markets like oil. In order to have spikes being independent of the volatility process $\Sigma(t)$ in the general model (1), we can switch off some of the $\omega_{j} \mathrm{~s}$ in (4), that is, choose some $\omega_{j}=0$. Then the $L_{i} \mathrm{~s}$ from the corresponding $\widetilde{L}_{j} \mathrm{~s}$ will give rise to independent spike components.

In electricity markets one observes spikes in the spot price dynamics (see, e.g. [13]). These spikes often occur seasonally. In the Nordic electricity market Nord-Pool, price spikes occur in the winter time when demand is high. We may therefore wish the jump frequency of the subordinators $L_{i}, i=1, \ldots, m$, to be time dependent. This is not possible when working with Lévy processes, but we can generalize to independent increment processes instead (see [22]). Independent increment processes can be thought of as time-inhomogeneous Lévy processes. Our modeling and analysis to come are easily modified to include such processes. To keep matters slightly more simplified, we restrict attention to the time-homogeneous case here. The interested reader is referred to [13] for applications of independent increment processes in energy markets.

We assume the following integrability conditions for the subordinators. It holds that

$$
\mathbb{E}\left[\log ^{+}\left\|\tilde{L}_{j}(1)\right\|\right]<\infty
$$

where $\log ^{+}(x)$ is defined as $\max (\log (x), 0), j=1, \ldots, n$, and $\|A\|^{2}=\operatorname{tr}\left(A^{\top} A\right)$ is the Frobenius norm of the matrix $A \in M_{d}(\mathbb{R})$. Note that this condition implies that

$$
\mathbb{E}\left[\log ^{+}\left|L_{i}(1)\right|\right]<\infty
$$

for $i=1, \ldots, m$, where $|\cdot|$ is the Euclidean 2-norm on $\mathbb{R}^{d}$.

In the next section we study the probabilistic properties of the factor processes $X$ and $Y_{i}$. As we will see, the analysis of the spot price model will depend crucially on the properties of certain operators, which will reflect the conditions on the matrices $A, B_{i}$, and $C_{j}$ for $i=1, \ldots, m$ and $j=1, \ldots, n$. Throughout the rest of the paper, we suppose that $\boldsymbol{A}, \boldsymbol{B}_{i}$, and $\boldsymbol{C}_{j}$ are invertible for $i=1, \ldots, m$ and $j=1, \ldots, n$. Furthermore, the matrices $A$ and $C_{j}$ are commuting for each $j=1, \ldots, n$. Finally, the operators $\boldsymbol{A}-\boldsymbol{C}_{j}$ are invertible for $j=1, \ldots, n$.

\section{Stationarity and probabilistic properties of the factor processes}

The processes $Y_{i}$ are Ornstein-Uhlenbeck processes, while $X$ is a volatility-modulated Ornstein-Uhlenbeck process. Applying the multidimensional Itô formula (see [21]) to the stochastic differential equations yields the following solutions: for $0 \leq s \leq t$,

$$
\begin{aligned}
& X(t)=\mathrm{e}^{A(t-s)} X(s)+\int_{s}^{t} \mathrm{e}^{A(t-u)} \Sigma(u)^{1 / 2} \mathrm{~d} W(u), \\
& Y_{i}(t)=\mathrm{e}^{B_{i}(t-s)} Y_{i}(s)+B_{i}^{-1}\left(I-\mathrm{e}^{B_{i}(t-s)}\right) \mu_{i}+\int_{s}^{t} \mathrm{e}^{B_{i}(t-u)} \eta_{i} \mathrm{~d} L_{i}(u)
\end{aligned}
$$

for $i=1, \ldots, m$. The matrix exponentials are defined as usual as $\mathrm{e}^{A}:=I+\sum_{k=1}^{\infty} A^{k} / k !$. 
According to Barndorff-Nielsen and Stelzer [7, Section 4], the solution of $Z_{j}(t), j=$ $1, \ldots, n$, is given by

$$
Z_{j}(t)=\mathrm{e}^{C_{j}(t-s)} Z_{j}(s) \mathrm{e}^{C_{j}^{\top}(t-s)}+\int_{s}^{t} \mathrm{e}^{C_{j}(t-u)} \mathrm{d} \widetilde{L}_{j}(u) \mathrm{e}^{C_{j}^{\top}(t-u)} .
$$

The matrix-valued stochastic integral in the second term of $Z_{j}(t)$ is understood as follows. For two $M_{d}(\mathbb{R})$-valued, bounded, and measurable functions $E(u)$ and $F(u)$ on $[t, s]$, the notation $\int_{s}^{t} E(u) \mathrm{d} \widetilde{L}(u) F(u)$ means the matrix $G(s, t) \in M_{d}(\mathbb{R})$ with coordinates defined by

$$
G_{i j}(s, t)=\sum_{k=1}^{d} \sum_{l=1}^{d} \int_{s}^{t} E_{i k}(u) F_{l j}(u) \mathrm{d} \tilde{L}_{k l}(u) .
$$

Here, $\widetilde{L}$ is the generic notation for some $\widetilde{L}_{j}$. We note that, since the $\widetilde{L}_{j}$ are supposed to be RCLL, the processes $Z_{j}$ also are RCLL.

Let us first look at the expected values of $X$ and $Y_{i}$. For this, we apply a slight generalization of the standard compensation formula for Lévy processes, stated and proven here for the convenience of the reader.

Lemma 1. Let L be an integrable Lévy process in $\mathbb{R}^{d}$, and let $f$ be a bounded measurable function from $[s, t]$ into $M_{d}(\mathbb{R})$ being of bounded variation. Then it holds that

$$
\mathbb{E}\left[\int_{s}^{t} f(u) \mathrm{d} L(u)\right]=\int_{s}^{t} f(u) \mathrm{d} u \mathbb{E}[L(1)] .
$$

Proof. Define the Lévy process $\widehat{L}(u):=L(u)-\mathbb{E}[L(1)] u$, which has expectation zero. From integration by parts (use the multidimensional Itô formula for jump processes in [21]), it holds that

$$
\int_{s}^{t} f(u) \mathrm{d} \widehat{L}(u)=f(t) \widehat{L}(t)-f(s) \widehat{L}(s)-\int_{s}^{t} \widehat{L}(u) \mathrm{d} f(u) .
$$

Now, choosing the RCLL version of $L$ (as we always can do for Lévy processes), we can apply the Fubini-Tonelli theorem to conclude that

$$
\mathbb{E}\left[\int_{s}^{t} f(u) \mathrm{d} \widehat{L}(u)\right]=0,
$$

and hence the lemma follows.

We find the following conditional expectations for the factor processes.

Lemma 2. Suppose that $\widetilde{L}_{j}(1)$ is integrable for $j=1, \ldots, n$. Then it holds that

$$
\begin{aligned}
\mathbb{E}\left[X(t) \mid \mathcal{F}_{s}\right] & =\mathrm{e}^{A(t-s)} X(s), \\
\mathbb{E}\left[Y_{i}(t) \mid \mathcal{F}_{s}\right] & =\mathrm{e}^{B_{i}(t-s)} Y_{i}(s)+B_{i}^{-1}\left(I-\mathrm{e}^{B_{i}(t-s)}\right) \mu_{i}+B_{i}^{-1}\left(\eta_{i}-\mathrm{e}^{B_{i}(t-s)} \eta_{i}\right) \mathbb{E}\left[L_{i}(1)\right]
\end{aligned}
$$

for $i=1, \ldots, m$.

Proof. By integrability of the subordinators $\widetilde{L}_{j}, \Sigma$ becomes integrable. The conditional expectation of $X(t)$ is thus given by

$$
\mathbb{E}\left[X(t) \mid \mathcal{F}_{s}\right]=\mathrm{e}^{A(t-s)} X(s)+\mathbb{E}\left[\int_{s}^{t} \mathrm{e}^{A(t-u)} \Sigma(u)^{1 / 2} \mathrm{~d} W(u)\right]=\mathrm{e}^{A(t-s)} X(s),
$$

since the Itô integral has zero expectation. 
For $Y_{i}, i=1, \ldots, m$, we obtain

$$
\begin{aligned}
\mathbb{E}\left[Y_{i}(t) \mid \mathcal{F}_{s}\right] & =\mathrm{e}^{B_{i}(t-s)} Y_{i}(s)+B_{i}^{-1}\left(I-\mathrm{e}^{B_{i}(t-s)}\right) \mu_{i}+\mathbb{E}\left[\int_{s}^{t} \mathrm{e}^{B_{i}(t-u)} \eta_{i} \mathrm{~d} L_{i}(u) \mid \mathcal{F}_{s}\right] \\
& =\mathrm{e}^{B_{i}(t-s)} Y_{i}(s)+B_{i}^{-1}\left(I-\mathrm{e}^{B_{i}(t-s)}\right) \mu_{i}+\int_{s}^{t} \mathrm{e}^{B_{i}(t-u)} \eta_{i} \mathrm{~d} u \mathbb{E}\left[L_{i}(1)\right] \\
& =\mathrm{e}^{B_{i}(t-s)} Y_{i}(s)+B_{i}^{-1}\left(I-\mathrm{e}^{B_{i}(t-s)}\right) \mu_{i}+B_{i}^{-1}\left(\eta_{i}-\mathrm{e}^{B_{i}(t-s)} \eta_{i}\right) \mathbb{E}\left[L_{i}(1)\right],
\end{aligned}
$$

where we have used Lemma 1 to obtain the last equality.

Since $A$ and $B_{i}$ have eigenvalues with negative real part, letting $t$ tend to $\infty$ yields

$$
\begin{gathered}
\lim _{t \rightarrow \infty} \mathbb{E}\left[X(t) \mid \mathcal{F}_{s}\right]=0, \\
\lim _{t \rightarrow \infty} \mathbb{E}\left[Y_{i}(t) \mid \mathcal{F}_{s}\right]=B_{i}^{-1}\left(\mu_{i}+\eta_{i} \mathbb{E}\left[L_{i}(1)\right]\right)
\end{gathered}
$$

for $i=1, \ldots, m$. Hence, in the limit, the 'base term' $X(t)$ will contribute zero in expectation, whereas the 'leverage terms' $Y_{i}$ will give a drift imposed from the subordinators and the coefficients $\mu_{i}$.

Let us analyse the second-order properties of the factor processes. We have the following result for the variance of the 'base component' $X(t)$.

Lemma 3. Assume that $\widetilde{L}_{j}(1)$ is integrable for $j=1, \ldots, n$. Then it holds that

$$
\begin{aligned}
\operatorname{var}\left[X(t) \mid \mathcal{F}_{s}\right]= & \sum_{j=1}^{n} \omega_{j}\left(\boldsymbol{A}-\boldsymbol{C}_{j}\right)^{-1}\left\{\mathrm{e}^{A(t-s)} Z_{j}(s) \mathrm{e}^{A^{\top}(t-s)}-\mathrm{e}^{C_{j}(t-s)} Z_{j}(s) \mathrm{e}^{C_{j}^{\top}(t-s)}\right\} \\
& +\sum_{j=1}^{n} \omega_{j} \boldsymbol{C}_{j}^{-1}\left\{( \boldsymbol { A } - \boldsymbol { C } _ { j } ) ^ { - 1 } \left\{\mathrm{e}^{A(t-s)} \mathbb{E}\left[\widetilde{L}_{j}(1)\right] \mathrm{e}^{A^{\top}(t-s)}\right.\right. \\
& \left.\left.-\mathrm{e}^{C_{j}(t-s)} \mathbb{E}\left[\widetilde{L}_{j}(1)\right] \mathrm{e}^{C_{j}^{\top}(t-s)}\right\}\right\} \\
& -\sum_{j=1}^{n} \omega_{j} \boldsymbol{A}^{-1}\left\{\boldsymbol{C}_{j}^{-1}\left\{\mathrm{e}^{A(t-s)} \mathbb{E}\left[\widetilde{L}_{j}(1)\right] \mathrm{e}^{A^{\top}(t-s)}-\mathbb{E}\left[\widetilde{L}_{j}(1)\right]\right\}\right\}
\end{aligned}
$$

for $0 \leq s \leq t$.

Proof. We compute the conditional variance for the process $X$ by appealing to the tower property of conditional expectations and the independent increment property of Brownian motion. Letting $g_{s, t}$ be the $\sigma$-algebra generated by $\mathcal{F}_{s}$ and the paths $\Sigma(u), s \leq u \leq t$, we find that

$$
\begin{aligned}
\operatorname{var}\left[X(t) \mid \mathcal{F}_{s}\right] & =\mathbb{E}\left[\left(\mathrm{e}^{A(t-s)} X(s)+\int_{s}^{t} \mathrm{e}^{A(s-u)} \Sigma(u)^{1 / 2} \mathrm{~d} W(u)\right)^{2} \mid \mathcal{F}_{s}\right]-\mathbb{E}\left[X(t) \mid \mathcal{F}_{s}\right]^{2} \\
& =\mathbb{E}\left[\mathbb{E}\left[\left(\int_{s}^{t} \mathrm{e}^{A(s-u)} \Sigma(u)^{1 / 2} \mathrm{~d} W(u)\right)^{2} \mid \mathcal{G}_{s, t}\right] \mid \mathcal{F}_{s}\right] \\
& =\mathbb{E}\left[\int_{s}^{t} \mathrm{e}^{A(t-u)} \Sigma(u) \mathrm{e}^{A^{\top}(t-u)} \mathrm{d} u \mid \mathcal{F}_{s}\right] \\
& =\sum_{j=1}^{n} \omega_{j} \int_{s}^{t} \mathrm{e}^{A(t-u)} \mathbb{E}\left[Z_{j}(u) \mid \mathcal{F}_{S}\right] \mathrm{e}^{A^{\top}(t-u)} \mathrm{d} u
\end{aligned}
$$


after appealing to Fubini's theorem. From the explicit representation of $Z_{j}(t)$ in (8), we find that

$$
\begin{aligned}
\mathbb{E}\left[Z_{j}(u) \mid \mathcal{F}_{s}\right] & =\mathrm{e}^{C_{j}(u-s)} Z_{j}(s) \mathrm{e}^{C_{j}^{\top}(u-s)}+\int_{s}^{u} \mathrm{e}^{C_{j}(u-v)} \mathbb{E}\left[\widetilde{L}_{j}(1)\right] \mathrm{e}^{C_{j}^{\top}(u-v)} \mathrm{d} v \\
& =\mathrm{e}^{C_{j}(u-s)} Z_{j}(s) \mathrm{e}^{C_{j}^{\top}(u-s)}+\int_{0}^{u-s} \mathrm{e}^{C_{j} z} \mathbb{E}\left[\widetilde{L}_{j}(1)\right] \mathrm{e}^{C_{j}^{\top} z} \mathrm{~d} z \\
& =\mathrm{e}^{C_{j}(u-s)} Z_{j}(s) \mathrm{e}^{C_{j}^{\top}(u-s)}+C_{j}^{-1}\left\{\mathrm{e}^{C_{j}(t-s)} \mathbb{E}\left[\widetilde{L}_{j}(1)\right] \mathrm{e}^{C_{j}^{\top}(t-s)}-\mathbb{E}\left[\widetilde{L}_{j}(1)\right]\right\},
\end{aligned}
$$

after appealing to Lemma 1 . Hence, using the fact that $A$ and $C_{j}$ are commuting for each $j=1, \ldots, n$, we find that

$$
\begin{aligned}
\operatorname{var}\left[X(t) \mid \mathcal{F}_{s}\right]= & \sum_{j=1}^{n} \omega_{j} \int_{s}^{t} \mathrm{e}^{A(t-u)} \mathrm{e}^{C_{j}(u-s)} Z_{j}(s) \mathrm{e}^{C_{j}^{\top}(u-s)} \mathrm{e}^{A^{\top}(t-u)} \mathrm{d} u \\
& +\sum_{j=1}^{n} \omega_{j} \boldsymbol{C}_{j}\left\{\int_{s}^{t} \mathrm{e}^{A(t-u)} \mathrm{e}^{C_{j}(u-s)} \mathbb{E}\left[\widetilde{L}_{j}(1)\right] \mathrm{e}^{C_{j}^{\top}(u-s)} \mathrm{e}^{A^{\top}(t-u)} \mathrm{d} u\right\} \\
& -\sum_{j=1} \omega_{j} \boldsymbol{C}_{j}^{-1} \int_{s}^{t} \mathrm{e}^{A(t-u)} \mathbb{E}\left[\widetilde{L}_{j}(1)\right] \mathrm{e}^{A^{\top}(t-u)} \mathrm{d} u \\
= & \sum_{j=1}^{n} \omega_{j}\left(\boldsymbol{A}-\boldsymbol{C}_{j}\right)^{-1}\left\{\mathrm{e}^{A(t-s)} Z_{j}(s) \mathrm{e}^{A^{\top}(t-s)}-\mathrm{e}^{C_{j}(t-s)} Z_{j}(s) \mathrm{e}^{C_{j}^{\top}(t-s)}\right\} \\
& +\sum_{j=1}^{n} \omega_{j} \boldsymbol{C}_{j}^{-1}\left\{( \boldsymbol { A } - \boldsymbol { C } _ { j } ) ^ { - 1 } \left\{\mathrm{e}^{A(t-s)} \mathbb{E}\left[\widetilde{L}_{j}(1)\right] \mathrm{e}^{A^{\top}(t-s)}\right.\right. \\
& -\sum_{j=1}^{n} \omega_{j} \boldsymbol{A}^{-1}\left\{\boldsymbol{C}_{j}^{-1}\left\{\mathrm{e}^{A(t-s)} \mathbb{E}\left[\widetilde{L}_{j}(1)\right] \mathrm{e}^{A^{\top}(t-s)} \mathbb{E}\left[\widetilde{L}_{j}(1)\right] \mathrm{e}^{C_{j}^{\top}(t-s)}\right\}\right\} \\
& \left.\left.\mathbb{E}\left[\widetilde{L}_{j}(1)\right]\right\}\right\} .
\end{aligned}
$$

The lemma follows.

Note that the explicit expression for the variance of the base component is computed under the condition that the matrices $A$ and $C_{j}$ are commutable. Moreover, we observe that, for Lemma 3 to hold, we must have the imposed conditions of invertibility of the operators $\boldsymbol{A}, \boldsymbol{C}_{j}$, and $\boldsymbol{A}-\boldsymbol{C}_{j}$. Recalling that the matrices $A$ and $C_{j}$ have eigenvalues with negative real part, we pass to the limit $t \rightarrow \infty$ to find that

$$
\lim _{t \rightarrow \infty} \operatorname{var}[X(t)]=\sum_{j=1}^{n} \omega_{j} \boldsymbol{A}^{-1} \boldsymbol{C}_{j}^{-1} \mathbb{E}\left[\widetilde{L}_{j}(1)\right] .
$$

Observe that the limit of the variance depends explicitly on the mean-reversion coefficient matrices $A$ and $C_{j}$. In fact, from Barndorff-Nielsen and Stelzer [7] we know that the stationary expected value of $Z_{j}(s)$ is $C_{j}^{-1} \mathbb{E}\left[\widetilde{L}_{j}(1)\right]$, so we can write

$$
\lim _{t \rightarrow \infty} \operatorname{var}\left[X(t) \mid \mathcal{F}_{s}\right]=A^{-1} \lim _{t \rightarrow \infty} \mathbb{E}[\Sigma(t)]
$$

for the limiting variance of the base component. This is in direct analogy with the univariate case (see [8]). 
We move on and find the variance of $Y_{i}(t)$.

Lemma 4. Suppose that $L_{i}(1)$ is square integrable for $i=1, \ldots, m$. Then it holds that

$$
\begin{aligned}
\operatorname{var}\left[Y_{i}(t) \mid \mathcal{F}_{s}\right]= & \boldsymbol{B}_{i}^{-1}\left(\eta_{i} \mathbb{E}\left[L_{i}(1) L_{i}^{\top}(1)\right] \eta_{i}^{\top}-\mathrm{e}^{B_{i}(t-s)} \eta_{i} \mathbb{E}\left[L_{i}(1) L_{i}^{\top}(1)\right] \eta_{i}^{\top} \mathrm{e}^{B_{i}^{\top}(t-s)}\right) \\
& -B_{i}^{-1}\left(I-\mathrm{e}^{B_{i}(t-s)}\right) \eta_{i} \mathbb{E}\left[L_{i}(1)\right] \mathbb{E}\left[L_{i}^{\top}(1)\right] \eta_{i}^{\top}\left(I-\mathrm{e}^{B_{i}^{\top}(t-s)}\right)\left(B_{i}^{-1}\right)^{\top}
\end{aligned}
$$

for $i=1, \ldots, m$ and $0 \leq s \leq t$.

Proof. Fix an $i=1, \ldots, n$. By (7) we find that the conditional variance of $Y_{i}(t)$ given $\mathcal{F}_{S}$ is

$$
\operatorname{var}\left[Y_{i}(t) \mid \mathcal{F}_{S}\right]=\operatorname{var}\left[\int_{s}^{t} \mathrm{e}^{B_{i}(t-u)} \eta_{i} \mathrm{~d} L_{i}(u) \mid \mathcal{F}_{S}\right] .
$$

Moreover, by the independent increment property of Lévy processes, it holds that

$$
\operatorname{var}\left[Y_{i}(t) \mid \mathcal{F}_{S}\right]=\operatorname{var}\left[\int_{s}^{t} \mathrm{e}^{B_{i}(t-u)} \eta_{i} \mathrm{~d} L_{i}(u)\right] .
$$

But, by the Itô isometry for Lévy process integrals,

$$
\begin{aligned}
\mathbb{E}\left[\int_{s}^{t}\right. & \left.\mathrm{e}^{B_{i}(t-u)} \eta_{i} \mathrm{~d} L_{i}(u) \int_{s}^{t} \mathrm{e}^{B_{i}(t-u)} \eta_{i} \mathrm{~d} L_{i}(u)^{\top}\right] \\
\quad= & \int_{s}^{t} \mathrm{e}^{B_{i}(t-u)} \eta_{i} \mathbb{E}\left[L_{i}(1) L_{i}^{\top}(1)\right] \eta_{i}^{\top} \mathrm{e}^{B_{i}^{\top}(t-u)} \mathrm{d} u \\
& =\boldsymbol{B}_{i}^{-1}\left(\eta_{i} \mathbb{E}\left[L_{i}(1) L_{i}^{\top}(1)\right] \eta_{i}^{\top}-\mathrm{e}^{B_{i}(t-s)} \eta_{i} \mathbb{E}\left[L_{i}(1) L_{i}^{\top}(1)\right] \eta_{i}^{\top} \mathrm{e}^{B_{i}^{\top}(t-s)}\right) .
\end{aligned}
$$

On the other hand, following from Lemma 1 ,

$$
\mathbb{E}\left[\int_{s}^{t} \mathrm{e}^{B_{i}(t-u)} \eta_{i} \mathrm{~d} L_{i}(u)\right]=\int_{s}^{t} \mathrm{e}^{B_{i}(t-u)} \mathrm{d} u \eta_{i} \mathbb{E}\left[L_{i}(1)\right]=B_{i}^{-1}\left(I-\mathrm{e}^{B_{i}(t-s)}\right) \eta_{i} \mathbb{E}\left[L_{i}(1)\right] .
$$

This completes the proof.

Note that we have used the standing condition of invertibility of the operators $\boldsymbol{B}_{i}$ in Lemma 4. For $Y_{i}(t)$, we can also compute an explicit limit for the variance using the fact that the eigenvalues of $B_{i}$ have negative real parts:

$$
\lim _{t \rightarrow \infty} \operatorname{var}\left[Y_{i}(t) \mid \mathcal{F}_{s}\right]=\boldsymbol{B}_{i}^{-1} \eta_{i} \mathbb{E}\left[L_{i}(1) L_{i}^{\top}(1)\right] \eta_{i}^{\top}-B_{i}^{-1} \eta_{i} \mathbb{E}\left[L_{i}(1)\right] \mathbb{E}\left[L_{i}^{\top}(1)\right] \eta_{i}^{\top}\left(B_{i}^{-1}\right)^{\top} .
$$

This holds for every $i=1, \ldots, m$.

From an empirical point of view, the covariance structures between factors and in the temporal direction are useful. We compute this in the next lemma.

Lemma 5. Suppose that $\widetilde{L}_{j}(1)$ is integrable for $j=1, \ldots, n$ and that $L_{i}(1)$ is square integrable for $i=1, \ldots, m$. Then, for $0 \leq s \leq t$,

$$
\operatorname{cov}\left[X(t), Y_{i}(t) \mid \mathcal{F}_{s}\right]=0=\operatorname{cov}\left[Y_{i}(t), Y_{j}(t) \mid \mathcal{F}_{s}\right]
$$

for $i \neq j$ and $i, j=1, \ldots, m$. Furthermore, the conditional autocovariance functions of $X$ and $Y_{i}$ are given by

$$
\begin{aligned}
& \operatorname{acov}_{X}(s, t, h):=\operatorname{cov}\left[X(t+h), X(t) \mid \mathcal{F}_{S}\right]=\mathrm{e}^{A h} \operatorname{var}\left[X(t) \mid \mathcal{F}_{s}\right], \\
& \operatorname{acov}_{Y_{i}}(s, t, h):=\operatorname{cov}\left[Y_{i}(t+h), Y_{i}(t) \mid \mathcal{F}_{s}\right]=\mathrm{e}^{B_{i} h} \operatorname{var}\left[Y_{i}(t) \mid \mathcal{F}_{s}\right]
\end{aligned}
$$

for $i=1, \ldots, m, 0 \leq s \leq t$, and $h \geq 0$ a constant (the lag of the autocovariance). 
Proof. First, from (7) we find that

$$
\operatorname{cov}\left[Y_{i}(t), Y_{j}(t) \mid \mathcal{F}_{s}\right]=\operatorname{cov}\left[\int_{s}^{t} \mathrm{e}^{B_{i}(t-u)} \eta_{i} \mathrm{~d} L_{i}(u), \int_{s}^{t} \mathrm{e}^{B_{j}(t-u)} \eta_{j} \mathrm{~d} L_{j}(u)\right]=0
$$

for $i \neq j$, since in that case $L_{i}$ and $L_{j}$ are independent.

Next, from (6) and (7) we find that, for given $i=1, \ldots, m$,

$$
\operatorname{cov}\left[X(t), Y_{i}(t) \mid \mathcal{F}_{s}\right]=\operatorname{cov}\left[\int_{s}^{t} \mathrm{e}^{A(t-u)} \Sigma(u)^{1 / 2} \mathrm{~d} W(u), \int_{s}^{t} \mathrm{e}^{B_{i}(t-u)} \eta_{i} \mathrm{~d} L_{i}(u) \mid \mathcal{F}_{s}\right] .
$$

We recall that $\Sigma(t)$ and $W(t)$ are independent. Using the tower property of conditional expectations, where we condition on the $\sigma$-algebra $g_{t, s}$ generated by all paths of $\widetilde{L}_{j}(u)$, $0 \leq u \leq t$, and $\mathcal{F}_{s}$, for $j=1, \ldots, n$, we find that

$$
\begin{aligned}
& \mathbb{E}\left[\left(\int_{s}^{t} \mathrm{e}^{A(t-u)} \Sigma(u)^{1 / 2} \mathrm{~d} W(u)\right)\left(\int_{s}^{t} \mathrm{e}^{B_{i}(t-u)} \eta_{i} \mathrm{~d} L_{i}(u)\right)^{\top} \mid \mathcal{F}_{S}\right] \\
& \quad=\mathbb{E}\left[\mathbb{E}\left[\left(\int_{s}^{t} \mathrm{e}^{A(t-u)} \Sigma(u)^{1 / 2} \mathrm{~d} W(u)\right)\left(\int_{s}^{t} \mathrm{e}^{B_{i}(t-u)} \eta_{i} \mathrm{~d} L_{i}(u)\right)^{\top} \mid g_{t, s}\right] \mid \mathcal{F}_{s}\right] \\
& \quad=\mathbb{E}\left[\mathbb{E}\left[\left(\int_{s}^{t} \mathrm{e}^{A(t-u)} \Sigma(u)^{1 / 2} \mathrm{~d} W(u)\right) \mid g_{t, s}\right]\left(\int_{s}^{t} \mathrm{e}^{B_{i}(t-u)} \eta_{i} \mathrm{~d} L_{i}(u)\right)^{\top} \mid \mathcal{F}_{s}\right] \\
& \quad=0 .
\end{aligned}
$$

In the second equality we have used the fact that the $L_{i}(u)$ for $i=1, \ldots, m$ are the diagonals of $\widetilde{L}_{i}(u)$, and, thus, measurable with respect to $g_{t, s}$, while the last equality follows since the expectation of an Itô integral is 0 .

Next, let us derive the autocovariance function for $X$. From (6), we find that, for $h \geq 0$,

$$
X(t+h)=\mathrm{e}^{A h} X(t)+\int_{t}^{t+h} \mathrm{e}^{A(t+h-u)} \Sigma(u)^{1 / 2} \mathrm{~d} W(u) .
$$

Hence,

$$
\operatorname{acov}_{X}(s, t, h)=\mathrm{e}^{A h} \operatorname{var}\left[X(t) \mid \mathcal{F}_{s}\right]+\operatorname{cov}\left[\int_{t}^{t+h} \mathrm{e}^{A(t+h-u)} \Sigma(u)^{1 / 2} \mathrm{~d} W(u), X(t) \mid \mathcal{F}_{S}\right] .
$$

By using the same double conditioning argument as above, we see that the second term is equal to 0 since Brownian motion has independent increments. This yields the autocovariance function of $X$. For the case $Y_{i}$, we apply the same argument using (7) and the independent increment property of Lévy processes to reach the result. This completes the proof.

From an empirical point of view, the limit of the autocovariance functions as $t \rightarrow \infty$ are particularly interesting. From (10) and (11), we have

$$
\begin{gathered}
\lim _{t \rightarrow \infty} \operatorname{acov}_{X}(s, t, h)=\mathrm{e}^{A h} \sum_{j=1}^{n} \omega_{j} \boldsymbol{A}^{-1} \boldsymbol{C}_{j}^{-1} \mathbb{E}\left[\widetilde{L}_{j}(1)\right], \\
\lim _{t \rightarrow \infty} \operatorname{acov}_{Y}(s, t, h)=\mathrm{e}^{B_{i} h}\left\{\boldsymbol{B}_{i}^{-1} \eta_{i} \mathbb{E}\left[L_{i}(1) L_{i}^{\top}(1)\right] \eta_{i}^{\top}-B_{i}^{-1} \eta_{i} \mathbb{E}\left[L_{i}(1)\right] \mathbb{E}\left[L_{i}^{\top}(1)\right] \eta_{i}^{\top}\right\} .
\end{gathered}
$$

As $A$ and $B_{i}$ have eigenvalues with negative real parts, we see that the deseasonalized log-spot prices $\ln S_{k}(t)-\ln \Lambda_{k}(t)$ of commodity $k=1, \ldots, d$ will in the limit have an autocorrelation 
function being a sum of exponential functions, with decay rates given by the real parts of the eigenvalues of $A$ and $B_{i}, i=1, \ldots, m$. This is an empirical feature we often see with energy prices (see, for example, [12]).

\subsection{Cumulants and stationary distributions}

Under the log-integrability conditions (5), the processes $Y_{i}$ and $Z_{j}$ converge to a stationary dynamic (see [28, Theorem 5.2]). In the next proposition the conditional characteristic function of the processes $X, Y_{i}$, and $Z_{j}$ for $i=1, \ldots, m$ and $j=1, \ldots, n$ are calculated in terms of the characteristic function of the matrix-valued processes $\widetilde{L}_{j}$.

Let us first investigate the cumulants of $Z_{j}$ and $Y_{i}, i=1, \ldots, m, j=1, \ldots, n$. To this end, we denote the conditional cumulant functions of $Y_{i}$ for $s \leq t$ by

$$
\phi_{Y_{i}}^{(s, t)}(z)=\ln \mathbb{E}\left[\mathrm{e}^{\mathrm{i} z^{\top} Y_{i}(t)} \mid \mathcal{F}_{s}\right], \quad z \in \mathbb{R}^{d},
$$

for $i=1, \ldots, m$. The conditional cumulant functions for $Z_{j}$ are

$$
\phi_{Z_{j}}^{(s, t)}(V)=\ln \mathbb{E}\left[\mathrm{e}^{\mathrm{itr}\left(V Z_{j}(t)\right)} \mid \mathcal{F}_{s}\right], \quad V \in M_{d}(\mathbb{R}),
$$

for $j=1, \ldots, n$. We have the following proposition for these conditional cumulants.

Proposition 1. For $t \geq s$, the conditional cumulant functions of $Y_{i}$ and $Z_{j}$ are respectively

$$
\begin{gathered}
\phi_{Y_{i}}^{(s, t)}(z)=\mathrm{i}\left(\mathrm{e}^{B_{i}(t-s)} Y_{i}(s)+\mathrm{i} B_{i}^{-1}\left(I-\mathrm{e}^{B_{i}(t-s)}\right) \mu_{i}\right)^{\top} z+\int_{0}^{t-s} \phi_{\widetilde{L}_{i}}\left(J_{d}\left(\eta_{i}^{\top} \mathrm{e}^{B_{i}^{\top} u} z\right)\right) \mathrm{d} u, \\
\phi_{Z_{j}}^{(s, t)}(V)=\operatorname{itr}\left(V \mathrm{e}^{C_{j}(t-s)} Z_{j}(s) \mathrm{e}^{C_{j}^{\top}(t-s)}\right)+\int_{0}^{t-s} \phi_{L_{j}}\left(\mathrm{e}^{C_{j}(t-s)} V \mathrm{e}^{C_{j}^{\top}(t-s)}\right) \mathrm{d} u
\end{gathered}
$$

for $i=1, \ldots, m$ and $j=1, \ldots, n$.

Proof. For the cumulants of $Y_{i}, i=1, \ldots, m$, we have, from (7),

$$
Y_{i}(t)=\mathrm{e}^{B_{i}(t-s)} Y^{i}(s)+B_{i}^{-1}\left(I-\mathrm{e}^{B_{i}(t-s)}\right) \mu_{i}+\int_{s}^{t} \mathrm{e}^{B_{i}(t-u)} \eta_{i} \mathrm{~d} L_{i}(u)
$$

for $t \geq s$. Hence, by the key formula (see [28]), the conditional cumulant function of $Y_{i}(t)$ given $\widetilde{F}_{S}$ is

$$
\begin{aligned}
\phi_{Y_{i}}^{(s, t)}(z) & =\mathrm{i}\left(\mathrm{e}^{B_{i}(t-s)} Y^{i}(s)+\mathrm{i} B_{i}^{-1}\left(I-\mathrm{e}^{B_{i}(t-s)}\right) \mu^{i}\right)^{\top} z+\int_{s}^{t} \phi_{L_{i}}\left(\eta_{i}^{\top} \mathrm{e}^{B_{i}^{\top}(t-u)} z\right) \mathrm{d} u \\
& =\mathrm{i}\left(\mathrm{e}^{B_{i}(t-s)} Y_{i}(s)+\mathrm{i} B_{i}^{-1}\left(I-\mathrm{e}^{B_{i}(t-s)}\right) \mu_{i}\right)^{\top} z+\int_{0}^{t-s} \phi_{L_{i}}\left(J_{d}\left(\eta_{i}^{\top} \mathrm{e}^{B_{i}^{\top} u} z\right)\right) \mathrm{d} u .
\end{aligned}
$$

The cumulant functions of the $Z_{j}$ s are computed in [25] (see also Theorem 2.5 of [23]). We include the derivation here for the convenience of the reader. By (8) and the independent 
increment property of Lévy processes,

$$
\begin{aligned}
\ln \mathbb{E}\left[\mathrm{e}^{\mathrm{itr}\left(V Z_{j}(t)\right)} \mid \mathcal{F}_{s}\right]= & \operatorname{itr}\left(V \mathrm{e}^{C_{j}(t-s)} Z_{j}(s) \mathrm{e}^{C_{j}^{\top}(t-s)}\right) \\
& +\ln \mathbb{E}\left[\exp \left(\operatorname{itr}\left(V \int_{s}^{t} \mathrm{e}^{C_{j}(t-u)} \mathrm{d} \widetilde{L}_{j}(u) \mathrm{e}^{C_{j}^{\top}(t-u)}\right)\right) \mid \widetilde{F}_{s}\right] \\
= & \operatorname{itr}\left(V \mathrm{e}^{C_{j}(t-s)} Z_{j}(s) \mathrm{e}^{C_{j}^{\top}(t-s)}\right) \\
& +\ln \mathbb{E}\left[\exp \left(\operatorname{itr}\left(V \int_{s}^{t} \mathrm{e}^{C_{j}(t-u)} \mathrm{d} \widetilde{L}_{j}(u) \mathrm{e}^{C_{j}^{\top}(t-u)}\right)\right)\right] \\
= & \operatorname{itr}\left(V \mathrm{e}^{C_{j}(t-s)} Z_{j}(s) \mathrm{e}^{C_{j}^{\top}(t-s)}\right)+\int_{s}^{t} \phi_{\widetilde{L}_{j}}\left(\mathrm{e}^{C_{j}(t-u)} V \mathrm{e}^{C_{j}^{\top}(t-u)}\right) \mathrm{d} u .
\end{aligned}
$$

This completes the proof.

Since $L(t)$ has finite $\log$ moments and $\sigma\left(B_{i}\right) \subseteq(-\infty, 0)+\mathrm{i} \mathbb{R}_{+}$, the limit of $\phi_{Y_{i}}^{(s, t)}$ for $t \rightarrow \infty$ is well defined (see [28]) and given by

$$
\lim _{t \rightarrow \infty} \phi_{Y_{i}}^{(s, t)}(z):=\phi_{Y_{i}}(z)=\mathrm{i} \mu_{i}^{\top}\left(B_{i}^{\top}\right)^{-1} z+\int_{0}^{\infty} \phi_{\widetilde{L}_{i}}\left(J_{d}\left(\eta_{i}^{\top} \mathrm{e}^{B_{i}^{\top} u} z\right)\right) \mathrm{d} u, \quad z \in \mathbb{R}^{d},
$$

for $i=1, \ldots, m$. This is the cumulant function of the stationary distribution of $Y_{i}$. Similarly, we find the cumulant function of the stationary distribution of the $Z_{j}$ s to be

$$
\lim _{t \rightarrow \infty} \phi_{Z_{j}}^{(s, t)}(V):=\phi_{Z_{j}}(V)=\int_{0}^{\infty} \phi_{\widetilde{L}_{j}}\left(\mathrm{e}^{C_{j}^{\top} s} V \mathrm{e}^{C_{j} s}\right) \mathrm{d} s, \quad V \in M_{d}(\mathbb{R}),
$$

for $j=1, \ldots, n$.

Let us continue our analysis by deriving the cumulant function and characterizing the stationary distribution of the base component $X$. To this end, we define the family of linear operators $\mathcal{C}_{j}(t)$ :

$$
\mathcal{C}_{j}(t): X \mapsto \omega_{j}\left[\left(\boldsymbol{C}_{j}-\boldsymbol{A}\right)^{-1}\left(\mathrm{e}^{C_{j} t} X \mathrm{e}^{C_{j}^{\top} t}-\mathrm{e}^{A t} X \mathrm{e}^{A^{\top} t}\right)\right] .
$$

We note that a similar operator is defined in [25] (see also [23]). The following auxiliary result is useful.

Lemma 6. Define $f(s, t):=\int_{s}^{t} \mathrm{e}^{A(t-u)} \Sigma(u) \mathrm{e}^{A^{\top}(t-u)} \mathrm{d} u$. Then it holds that

$$
f(s, t)=\sum_{j=1}^{n} \mathcal{C}_{j}(t-s) Z_{j}(s)+\int_{s}^{t} \mathcal{C}_{j}(t-v) \mathrm{d} \widetilde{L}_{j}(v)
$$

for $0 \leq s \leq t$.

Proof. Using (8) and the assumption that $A$ and $C_{j}$ commute for $j=1, \ldots, n$, it holds that

$$
\begin{gathered}
f(s, t)=\int_{s}^{t} \mathrm{e}^{A(t-u)} \sum_{j=1}^{n} \omega_{j}\left(\mathrm{e}^{C_{j}(u-s)} Z_{j}(s) \mathrm{e}^{C_{j}^{\top}(u-s)}+\int_{s}^{u} \mathrm{e}^{C_{j}(u-v)} \mathrm{d} \widetilde{L}_{j}(v) \mathrm{e}^{C_{j}^{\top}(u-v)}\right) \\
\quad \times \mathrm{e}^{A^{\top}(t-u)} \mathrm{d} u
\end{gathered}
$$




$$
\begin{aligned}
& =\sum_{j=1}^{n} \omega_{j} \int_{s}^{t} \mathrm{e}^{\left(C_{j}-A\right) u} \mathrm{e}^{A t-C_{j} s}\left(Z_{j}(s)+\int_{s}^{u} \mathrm{e}^{-C_{j} v} \mathrm{~d} \widetilde{L}_{j}(v) \mathrm{e}^{-C_{j}^{\top} v}\right) \\
& \quad \times \mathrm{e}^{A^{\top} t-C_{j}^{\top} s} \mathrm{e}^{\left(C_{j}-A\right)^{\top} u} \mathrm{~d} u \\
& =\sum_{j=1}^{n} \omega_{j}\left(\boldsymbol{C}_{j}-\boldsymbol{A}\right)^{-1}\left(\mathrm{e}^{C_{j}(t-s)} Z_{j}(s) \mathrm{e}^{C_{j}^{\top}(t-s)}-\mathrm{e}^{A(t-s)} Z_{j}(s) \mathrm{e}^{A^{\top}(t-s)}\right) \\
& +\int_{s}^{t} \int_{s}^{u}\left\{\mathrm{e}^{\left(C_{j}-A\right) u} \mathrm{e}^{A t} \mathrm{e}^{-C_{j} v} \mathrm{~d} \widetilde{L}_{j}(v) \mathrm{e}^{-C_{j}^{\top} v} \mathrm{e}^{A^{\top} t} \mathrm{e}^{\left(C_{j}-A\right)^{\top} u}\right\} \mathrm{d} u .
\end{aligned}
$$

The last integral is interpreted as first integrating with respect to $\mathrm{d} \widetilde{L}_{j}(v)$, and then integrating the obtained expression with respect to $\mathrm{d} u$. But, by spelling out the integrals in terms of sums, using the definition of the $\mathrm{d} \widetilde{L}_{j}(v)$ integrals, and invoking the stochastic Fubini theorem (see [26]), we obtain

$$
\begin{aligned}
& \int_{s}^{t} \int_{s}^{u}\left\{\mathrm{e}^{\left(C_{j}-A\right) u} \mathrm{e}^{A t} \mathrm{e}^{-C_{j} v} \mathrm{~d} \widetilde{L}_{j}(v) \mathrm{e}^{-C_{j}^{\top} v} \mathrm{e}^{A^{\top} t} \mathrm{e}^{\left(C_{j}-A\right)^{\top} u}\right\} \mathrm{d} u \\
& \quad=\int_{s}^{t} \int_{v}^{t}\left\{\mathrm{e}^{\left(C_{j}-A\right) u} \mathrm{e}^{A t} \mathrm{e}^{-C_{j} v} \mathrm{~d} \widetilde{L}_{j}(v) \mathrm{e}^{-C_{j}^{\top} v} \mathrm{e}^{A^{\top} t} \mathrm{e}^{\left(C_{j}-A\right)^{\top} u}\right\} \mathrm{d} u .
\end{aligned}
$$

Here, the right-hand side is interpreted as first integrating with respect to $\mathrm{d} u$, and then integrating with respect to $\mathrm{d} \widetilde{L}_{j}(v)$. This is done by computing the matrix in the brackets, and then in each coordinate performing an integration first with respect to $\mathrm{d} u$ and then with respect to the relevant coordinates of $\mathrm{d} \widetilde{L}_{j}(v)$. Hence, we find that

$$
\begin{aligned}
f(s, t)= & \sum_{j=1}^{n} \mathcal{C}_{j}(t-s) Z_{j}(s) \\
& +\left(\boldsymbol{C}_{j}-\boldsymbol{A}\right)^{-1}\left(\int_{s}^{t} \mathrm{e}^{C_{j}(t-v)} \mathrm{d} \widetilde{L}_{j}(v) \mathrm{e}^{C_{j}^{\top}(t-v)}-\int_{s}^{t} \mathrm{e}^{A(t-v)} \mathrm{d} \widetilde{L}_{j}(v) \mathrm{e}^{A^{\top}(t-v)}\right) .
\end{aligned}
$$

This completes the proof.

With this result at hand, we can derive the conditional cumulant function of $X(t)$ defined by

$$
\phi_{X}^{(s, t)}(z)=\ln \mathbb{E}\left[\mathrm{e}^{\mathrm{i} z^{\top} X(t)} \mid \mathcal{F}_{s}\right], \quad z \in \mathbb{R}^{d},
$$

for $s \leq t$. This is done in the next proposition.

Proposition 2. The conditional cumulant function of the process $X(t)$ given $\mathcal{F}_{s}$ is

$$
\begin{aligned}
\phi_{X}^{(s, t)}(z)= & \mathrm{i} X^{\top}(s) \mathrm{e}^{A^{\top}(t-s)} z-\frac{1}{2} \sum_{j=1}^{n} z^{\top} \mathcal{C}_{j}(t-s) Z_{j}(s) z \\
& +\sum_{j=1}^{n} \int_{0}^{t-s} \phi_{\widetilde{L}_{j}}\left(\frac{1}{2} \mathrm{i} \mathcal{C}_{j}^{*}(u) z z^{\top}\right) \mathrm{d} u
\end{aligned}
$$

for every $0 \leq s \leq t$ and $z \in \mathbb{R}^{d}$, where $\mathcal{C}_{j}^{*}$ is the adjoint operator of $\mathcal{C}_{j}$ defined in (13). 
Proof. Let $g_{t, s}$ denote the filtration generated by $\mathcal{F}_{s}$ and the paths $\Sigma(u), 0 \leq u \leq t$. By the independence of $W$ and $\widetilde{L}_{j}$ for $j=1, \ldots, n$, and the tower property of conditional expectations, we have

$$
\begin{aligned}
\phi_{X}^{(s, t)}(z)= & \ln \mathbb{E}\left[\mathbb{E}\left[\mathrm{e}^{\mathrm{i} z z^{\top} X(t)} \mid g_{t, s}\right] \mid \mathcal{F}_{s}\right] \\
= & \mathrm{i} X^{\top}(s) \mathrm{e}^{A^{\top}(t-s)} z \\
& +\ln \mathbb{E}\left[\mathbb{E}\left[\exp \left(\mathrm{i}\left(\int_{s}^{t} \Sigma(u)^{1 / 2} \mathrm{e}^{A(t-u)} \mathrm{d} W(u)\right)^{\top} z\right) \mid g_{t, s}\right] \mid \mathcal{F}_{s}\right] \\
= & \mathrm{i} X^{\top}(s) \mathrm{e}^{A^{\top}(t-s)} z+\ln \mathbb{E}\left[\exp \left(-\frac{1}{2} z^{\top} \int_{s}^{t} \mathrm{e}^{A(t-u)} \Sigma(u) \mathrm{e}^{A^{\top}(t-u)} \mathrm{d} u z\right) \mid \mathcal{F}_{s}\right] .
\end{aligned}
$$

In the second equality we used (6) and in the third equality we used the Gaussianity of a Wiener integral (note that the integrand is a deterministic function after conditioning on $g_{t, s}$ ). Appealing to Lemma 6, we find that

$$
\begin{aligned}
\phi_{X}^{(s, t)}(z)= & \mathrm{i} X^{\top}(s) \mathrm{e}^{A^{\top}(t-s)} z-\frac{1}{2} \sum_{j=1}^{n} z^{\top} \mathcal{C}_{j}(t-s) Z_{j}(s) z \\
& +\sum_{j=1}^{n} \ln \mathbb{E}\left[\exp \left(-\frac{1}{2} z^{\top} \int_{s}^{t} \mathcal{C}_{j}(t-u) \mathrm{d} \widetilde{L}_{j}(u) z\right) \mid \mathcal{F}_{s}\right] \\
= & \mathrm{i} X^{\top}(s) \mathrm{e}^{A^{\top}(t-s)} z-\frac{1}{2} \sum_{j=1}^{n} z^{\top} \mathcal{C}_{j}(t-s) Z_{j}(s) z \\
& +\sum_{j=1}^{n} \ln \mathbb{E}\left[\exp \left(\mathrm{i} \operatorname{tr}\left(\frac{1}{2} \mathrm{i} z z^{\top} \int_{s}^{t} \mathcal{C}_{j}(t-u) \mathrm{d} \widetilde{L}_{j}(u)\right)\right)\right] .
\end{aligned}
$$

In the last step, we used the fundamental relation $z^{\top} U z=\operatorname{tr}\left(z z^{\top} U\right)$ for a quadratic matrix $U$ together with the independent increment property of Lévy processes. Now, observe that the stochastic integral can be expressed as

$$
\int_{s}^{t} \mathcal{C}_{j}(t-u) \mathrm{d} \widetilde{L}_{j}(u)=\lim _{\left|\Delta_{i}\right| \rightarrow 0} \sum_{i=0}^{n-1} \mathcal{C}_{j}\left(t-u_{i}\right) \Delta \widetilde{L}_{j}\left(u_{i}\right)
$$

for partitions $s=u_{0}<\cdots<u_{n}=t$ with

$$
\Delta \widetilde{L}_{j}\left(u_{i}\right):=\widetilde{L}_{j}\left(u_{i+1}\right)-\widetilde{L}_{j}\left(u_{i}\right) \quad \text { and } \quad \Delta_{i}:=u_{i+1}-u_{i} .
$$

By the independence of increments of a Lévy process, and continuity of the exponential function together with Fubini-Tonelli's theorem, we obtain

$$
\begin{aligned}
\ln \mathbb{E}\left[\exp \left(\mathrm{i} \operatorname{tr}\left(\frac{1}{2} \mathrm{i} z z^{\top} \int_{s}^{t} \mathcal{C}_{j}(t-u) \mathrm{d} \widetilde{L}_{j}(u)\right)\right)\right] \\
=\lim _{\left|\Delta_{i}\right| \rightarrow 0} \sum_{i=0}^{n-1} \ln \mathbb{E}\left[\exp \left(\mathrm{i} \operatorname{tr}\left(\frac{1}{2} \mathrm{i} z z^{\top} \mathcal{C}_{j}\left(t-u_{i}\right) \Delta \widetilde{L}_{j}\left(u_{i}\right)\right)\right)\right] .
\end{aligned}
$$

Now, the linear operators $\mathcal{C}_{j}\left(t-u_{i}\right)$ can be represented as vec ${ }^{-1} \circ \mathcal{K} \circ$ vec for a matrix $\mathcal{K} \in \mathbb{R}^{d^{2}}$. 
Hence, since for quadratic matrices $V$ it holds that $\operatorname{tr}(V X)=\operatorname{vec}(V)^{\top} \operatorname{vec}(X)$, we find that

$$
\begin{aligned}
\operatorname{tr}\left(V \mathcal{C}_{j}\left(t-u_{i}\right) \Delta \widetilde{L}_{j}\left(u_{i}\right)\right) & =\operatorname{vec}(V)^{\top} \operatorname{vec}\left(\mathcal{C}_{j}\left(t-u_{i}\right) \Delta \widetilde{L}_{j}\left(u_{i}\right)\right) \\
& =\operatorname{vec}(V)^{\top} \operatorname{vec}\left(\operatorname{vec}^{-1} \circ \mathcal{K} \circ \operatorname{vec}\left(\Delta \widetilde{L}_{j}\left(u_{i}\right)\right)\right) \\
& =\operatorname{vec}(V)^{\top} \mathcal{K} \operatorname{vec}\left(\Delta \widetilde{L}_{j}\left(u_{i}\right)\right) \\
& =\left(\mathcal{K}^{\top} \operatorname{vec}(V)\right)^{\top} \operatorname{vec}\left(\Delta \widetilde{L}_{j}\left(u_{i}\right)\right) .
\end{aligned}
$$

Thus,

$$
\begin{aligned}
\ln \mathbb{E}\left[\exp \left(i \operatorname{tr}\left(V \mathcal{C}_{j}\left(t-u_{i}\right) \Delta \widetilde{L}_{j}\left(u_{i}\right)\right)\right)\right] & =\ln \mathbb{E}\left[\exp \left(\mathrm{i}\left(\mathcal{K}^{\top} \operatorname{vec}(V)\right)^{\top} \operatorname{vec}\left(\Delta \widetilde{L}_{j}\left(u_{i}\right)\right)\right)\right] \\
& =\ln \mathbb{E}\left[\exp \left(i \operatorname{tr}\left(\operatorname{vec}^{-1}\left(\mathcal{K}^{\top} \operatorname{vec}(V)\right) \Delta \widetilde{L}_{j}\left(u_{i}\right)\right)\right)\right] \\
& =\phi_{\widetilde{L}_{j}}\left(\operatorname{vec}^{-1} \circ \mathcal{K}^{\top} \circ \operatorname{vec}(V)\right) \Delta_{i} \\
& =\phi_{\widetilde{L}_{j}}\left(\mathcal{C}_{j}^{*}\left(t-u_{i}\right) V\right) \Delta_{i}
\end{aligned}
$$

Letting $V=\frac{1}{2} \mathrm{i} z z^{\top}$, we conclude that

$$
\begin{aligned}
\lim _{\left|\Delta_{i}\right| \rightarrow 0} \sum_{i=0}^{n-1} \ln \mathbb{E}\left[\exp \left(\mathrm{i} \operatorname{tr}\left(\frac{1}{2} \mathrm{i} z z^{\top} \mathcal{C}_{j}\left(t-u_{i}\right) \Delta \widetilde{L}_{j}\left(u_{i}\right)\right)\right)\right] & =\int_{s}^{t} \phi_{\widetilde{L}_{j}}\left(\frac{1}{2} \mathrm{i} \mathcal{C}_{j}^{*}(t-u) z z^{\top}\right) \mathrm{d} u \\
& =\int_{0}^{t-s} \phi_{\widetilde{L}_{j}}\left(\frac{1}{2} \mathrm{i} \mathcal{C}_{j}^{*}(u) z z^{\top}\right) \mathrm{d} u
\end{aligned}
$$

This completes the proof.

We can prove that $X(t)$ converges to a stationary dynamic, and derive the cumulant function for the limiting distribution.

Proposition 3. The process $X(t)$ converges to a stationary dynamic, and the cumulant function of the limiting distribution is given by

$$
\lim _{t \rightarrow \infty} \phi_{X}^{(s, t)}(z):=\phi_{X}(z)=\sum_{j=1}^{n} \int_{0}^{\infty} \phi_{\widetilde{L}_{j}}\left(\frac{1}{2} \mathrm{i} \mathcal{C}_{j}^{*}(s) z z^{\top}\right) \mathrm{d} s,
$$

where $z \in \mathbb{R}^{d}$ and the linear operator $\mathcal{C}_{j}(t)$ is defined in (13).

Proof. By the definition of $\mathcal{C}_{j}(t)$ and the fact that $A$ and $C_{j}, j=1, \ldots, n$, have eigenvalues with negative real parts, it is straightforward to see that

$$
\lim _{t \rightarrow \infty} \mathrm{i} X^{\top}(s) \mathrm{e}^{A^{\top}(t-s)} z-\frac{1}{2} \sum_{j=1}^{n} z^{\top} \mathcal{C}_{j}(t-s) Z_{j}(s) z=0 .
$$

Hence, we must prove that the integral

$$
\int_{0}^{t} \phi_{\tilde{L}_{j}}\left(\frac{1}{2} \mathrm{i} \mathcal{C}_{j}^{*}(s) z z^{\top}\right) \mathrm{d} s
$$

converges when $t \rightarrow \infty$ for every $j=1, \ldots, n$. To prove this, it is sufficient to show that

$$
\int_{s}^{t} \mathcal{C}_{j}(t-u) \mathrm{d} \widetilde{L}_{j}(u)
$$


converges in distribution for each $j=1, \ldots, n$ as $t \rightarrow \infty$. Let us fix $j=1, \ldots, n$, and observe that, by the definition of $\mathcal{C}_{j}(t)$ and the linearity of the $\boldsymbol{C}_{j}-\boldsymbol{A}$ operator, we have

$$
\begin{aligned}
& \int_{s}^{t} \mathcal{C}_{j}(t-u) \mathrm{d} \widetilde{L}_{j}(u) \\
& \quad=\omega_{j}\left(\boldsymbol{C}_{j}-\boldsymbol{A}\right)^{-1}\left\{\int_{s}^{t} \mathrm{e}^{C_{j}(t-u)} \mathrm{d} \widetilde{L}_{j}(u) \mathrm{e}^{C_{j}(t-u)}-\int_{s}^{t} \mathrm{e}^{A(t-u)} \mathrm{d} \widetilde{L}_{j}(u) \mathrm{e}^{A(t-u)}\right\} .
\end{aligned}
$$

But, the two stochastic integrals converge in distribution when $t \rightarrow \infty$ for fixed $s$ by [28, Theorem 5.2], since $A$ and $C_{j}$ have eigenvalues with negative real parts. This completes the proof.

We observe that the limiting distribution of $X$ when $t \rightarrow \infty$ is centered and symmetric since its cumulant function satisfies $\phi_{X}(z)=\phi_{X}(-z)$. We discuss the stationary distribution of $X$ in more detail.

As we now argue, the stationary distribution of $X$ can be viewed as the convolution of a centered normal and a leptokurtic distribution whenever $\widetilde{L}_{j}(1)$ is integrable for $j=1, \ldots, n$. To this end, introduce the zero-mean matrix-valued Lévy process $\widehat{L}_{j}(t):=\widetilde{L}_{k}(t)-\mathbb{E}\left[\widetilde{L}_{j}(1)\right] t$, and denote by $\phi_{\widehat{L}_{j}}(V)$ its cumulant defined by

$$
\phi_{\widehat{L}_{j}}(V)=\phi_{\widetilde{L}_{j}}(V)-\mathrm{i} \operatorname{tr}\left(V \mathbb{E}\left[\widetilde{L}_{j}(1)\right]\right)
$$

The cumulant function of the stationary distribution of $X(t)$ can henceforth be expressed as

$$
\begin{aligned}
\phi_{X}(z) & =\sum_{j=1}^{n}\left\{\int_{0}^{\infty} \phi_{\widehat{L}_{j}}\left(\frac{1}{2} \mathrm{i} \mathcal{C}_{j}^{*}(s) z z^{\top}\right) \mathrm{d} s+\mathrm{i} \int_{0}^{\infty} \operatorname{tr}\left(\frac{1}{2} \mathrm{iC}_{j}^{*}(s) z z^{\top} \mathbb{E}\left[\widetilde{L}_{j}(1)\right]\right) \mathrm{d} s\right\} \\
& =\sum_{j=1}^{n}\left\{\int_{0}^{\infty} \phi_{\widehat{L}_{j}}\left(\frac{1}{2} \mathrm{i} \mathcal{C}_{j}^{*}(s) z z^{\top}\right) \mathrm{d} s-\frac{1}{2} \int_{0}^{\infty} \operatorname{tr}\left(\mathcal{C}_{j}^{*}(s) z z^{\top} \mathbb{E}\left[\widetilde{L}_{j}(1)\right]\right) \mathrm{d} s\right\}
\end{aligned}
$$

By the properties of the trace operator, we have

$$
\begin{aligned}
\operatorname{tr}\left(\left(\mathcal{C}_{j}^{*}(s) z z^{\top}\right) \mathbb{E}\left[\widetilde{L}_{j}(1)\right]\right) & =\operatorname{vec}\left(\mathcal{C}_{j}^{*}(s) z z^{\top}\right)^{\top} \operatorname{vec}\left(\mathbb{E}\left[\widetilde{L}_{j}(1)\right]\right) \\
& =\operatorname{vec}\left(\operatorname{vec}^{-1}\left(\mathcal{K}^{\top} \operatorname{vec}\left(z z^{\top}\right)\right)\right)^{\top} \operatorname{vec}\left(\mathbb{E}\left[\widetilde{L}_{j}(1)\right]\right) \\
& =\left(\mathcal{K}^{\top} \operatorname{vec}\left(z z^{\top}\right)\right)^{\top} \operatorname{vec}\left(\mathbb{E}\left[\widetilde{L}_{j}(1)\right]\right) \\
& =\operatorname{vec}\left(z z^{\top}\right) \mathcal{K} \operatorname{vec}\left(\mathbb{E}\left[\widetilde{L}_{j}(1)\right]\right) \\
& =\operatorname{tr}\left(z z^{\top} \operatorname{vec}^{-1}\left(\mathcal{K} \operatorname{vec}\left(\mathbb{E}\left[\widetilde{L}_{j}(1)\right]\right)\right)\right) \\
& =\operatorname{tr}\left(z z^{\top} \mathcal{C}_{j}(s) \mathbb{E}\left[\widetilde{L}_{j}(1)\right]\right) \\
& =z^{\top} \mathcal{C}_{j}(s) \mathbb{E}\left[\widetilde{L}_{j}(1)\right] z
\end{aligned}
$$

Here, we have used the fact that the operator $\mathcal{C}_{j}(s)$ can be represented by the $\mathbb{R}^{d^{2} \times d^{2}}$-matrix $\mathcal{K}$ as $\mathcal{C}_{j}(s)=\operatorname{vec}^{-1} \circ \mathcal{K} \circ$ vec. Using Lemma 3 , we conclude that

$$
\phi_{X}(z)=\sum_{j=1}^{n} \int_{0}^{\infty} \phi_{\widehat{L}_{j}}\left(\frac{1}{2} \mathrm{i} \mathcal{C}_{j}^{*}(s) z z^{\top}\right) \mathrm{d} s-\frac{1}{2} z^{\top}\left(\lim _{t \rightarrow \infty} \operatorname{var}[X(t)]\right) z .
$$


The last term is the characteristic function of a centered multivariate normal distribution with variance equal to $\lim _{t \rightarrow \infty} \operatorname{var}[X(t)]$. We note that this coincides with the stationary distribution obtained from the multivariate Schwartz model having constant volatility $\Sigma \in M_{d}(\mathbb{R})$ given by

$$
\Sigma:=\lim _{t \rightarrow \infty} \operatorname{var}[X(t)]
$$

The first term in $\phi_{X}(z)$ will be the characteristic function of a non-Gaussian distribution.

\section{Analysis of the spot dynamics}

Let us look at the dynamics of $\widetilde{S}(t):=S(t) / \Lambda(t)$, the deseasonalized spot price, where the division is done elementwise.

Proposition 4. It holds that

$$
\mathrm{d} \ln \widetilde{S}(t)=(M(t)+A \ln \widetilde{S}(t)) \mathrm{d} t+\Sigma(t)^{1 / 2} \mathrm{~d} W(t)+\sum_{i=1}^{m} \eta_{i} \mathrm{~d} L_{i}(t),
$$

where

$$
M(t)=\sum_{i=1}^{m} \mu_{i}+\left(-A+B_{j}\right) Y_{j}(t) .
$$

Proof. The proof follows from rewriting (2) and (3).

We see from this result that the dynamics can be interpreted as a mean-reverting process towards a stochastic mean. The mean will be described by the multivariate process $M(t)$, which will consist of linear combinations of the different 'spike' components $Y_{j}$. The matrix $A$ describes the 'speed' of mean reversion, as well as how the different commodities are functionally dependent on each other. Moreover, the stochastic volatility term and the spike contributions are clearly evident in the two last terms of the dynamics.

We move onto analyzing the limiting distribution of $\ln \widetilde{S}(t)$. From Lemma 2, we find that

$$
\lim _{t \rightarrow \infty} \mathbb{E}[\ln \widetilde{S}(t)]=\lim _{t \rightarrow \infty} \mathbb{E}[X(t)]+\sum_{i=1}^{m} \mathbb{E}\left[Y_{i}(t)\right]=\sum_{i=1}^{m} B_{i}^{-1}\left(\mu_{i}+\eta_{i} \mathbb{E}\left[L_{i}(1)\right]\right) .
$$

Furthermore, from Lemma 5, after passing to the limit as $t \rightarrow \infty$, the autocovariance function of $\ln \widetilde{S}(t)$ becomes

$$
\begin{aligned}
\operatorname{acov}_{\ln \widetilde{S}}(h)= & \operatorname{acov}_{X}(h)+\operatorname{acov}_{\sum} Y_{i}(h) \\
= & \mathrm{e}^{A|h|} \sum_{j=1}^{n} \omega_{j} \boldsymbol{A}^{-1} \boldsymbol{C}_{j}^{-1} \mathbb{E}\left[\widetilde{L}_{j}(1)\right] \\
& +\sum_{i=1}^{m} \mathrm{e}^{B_{i}|h|}\left(\boldsymbol{B}_{i}^{-1} \eta_{i} \mathbb{E}\left[L_{i}(1) L_{i}^{\top}(1)\right]\left(\eta_{i}\right)^{\top}-B_{i}^{-1} \eta_{i} \mathbb{E}\left[L_{i}(1)\right] \mathbb{E}\left[L_{i}^{\top}(1)\right] \eta_{i}^{\top}\right) .
\end{aligned}
$$

Hence, asymptotically, the autocovariance function of $\ln \widetilde{S}(t)$ will be a linear combination of exponentially decaying functions due to eigenvalues having a negative real part. Real eigenvalues will give pure exponentially decaying functions, while nonreal eigenvalues include a sinusoidal behavior. Furthermore, when the matrices are nondiagonalisable, we have a 
polynomial behavior to the exponential decay. In power markets, the empirical autocovariance functions seem to be well-represented as linear combinations of exponential functions (see, e.g. [12]).

By combining the results of the cumulant functions for the different factors in the dynamics of $\ln \widetilde{S}(t)$ derived in the previous section, we can compute the cumulant of the deseasonalized log-spot prices. This is presented in the next proposition.

Proposition 5. The logarithmic deseasonalized spot price $\ln \widetilde{S}(t)$ converges to a stationary dynamic with a limiting distribution having cumulant function given by

$$
\begin{aligned}
\phi_{\ln \widetilde{S}}(z)= & \sum_{i=1}^{m} \mathrm{i} \mu_{i}^{\top}\left(B_{i}^{\top}\right)^{-1} z+\sum_{j=1}^{n} \int_{0}^{\infty} \phi_{\tilde{L}_{j}}\left(\frac{1}{2} \mathrm{i} \mathcal{C}_{j}^{*}(u) z z^{\top}\right) \mathrm{d} u \\
& +\sum_{i=1}^{m} \int_{0}^{\infty}\left[\phi_{\widetilde{L}_{i}}\left(\frac{1}{2} \mathrm{i} \mathcal{C}_{i}^{*}(u) z z^{\top}+J_{d}\left(\eta_{i}^{\top} \mathrm{e}^{B_{i}^{\top} u} z\right)\right)-\phi_{\widetilde{L}_{i}}\left(\frac{1}{2} \mathrm{i} \mathcal{C}_{i}^{*}(u) z z^{\top}\right)\right] \mathrm{d} u
\end{aligned}
$$

for $z \in \mathbb{R}^{d}$.

Proof. By combining Proposition 2 and (12), the conditional cumulant function of $\ln \widetilde{S}$ given $\mathcal{F}_{S}$ is

$$
\begin{aligned}
\phi_{\ln \widetilde{S}}^{(s, t)}(z)= & \mathrm{i} X^{\top}(s) \mathrm{e}^{A^{\top}(t-s)} z-\frac{1}{2} \sum_{j=1}^{n} z^{\top} \mathcal{C}_{j}(t-s) Z_{j}(s) z \\
& +\sum_{i=1}^{m} \mathrm{i} Y^{\top}(s) \mathrm{e}^{B_{i}^{\top}(t-s)} z+\mathrm{i}\left(B_{i}^{-1}\left(I-\mathrm{e}^{B_{i}(t-s)}\right) \mu_{i}\right)^{\top} z \\
& +\sum_{i=1}^{m} \int_{0}^{t-s} \phi_{\widetilde{L}_{i}}\left(\frac{1}{2} \mathrm{i} \mathcal{C}_{i}^{*}(u) z z^{\top}+J_{d}\left(\eta_{i}^{\top} \mathrm{e}^{B_{i}^{\top} u} z\right)\right) \mathrm{d} u \\
& +\sum_{j=m+1}^{n} \int_{0}^{t-s} \phi_{\widetilde{L}_{j}}\left(\frac{1}{2} \mathrm{i} \mathcal{C}_{j}^{*}(u) z z^{\top}\right) \mathrm{d} u .
\end{aligned}
$$

Since a stationary limit exists for $X$ and all $Y_{i}$ s, there also exists a stationary limit for $\ln \widetilde{S}$. Proposition 5 follows by taking limits for $t \rightarrow \infty$ using the fact that the real parts of the eigenvalues of the involved matrices are negative.

Note that the sum over $j$ in the expression for $\phi_{\ln } \widetilde{S}$ stems from the stationary cumulant of $X$, and, therefore, is from a symmetric centered random variable. Stationarity is a desirable feature in commodity markets, being a reflection of supply and demand-driven prices. However, many researchers argue for nonstationary effects (as, for example, Burger et al. [15], who studied German electricity spot prices). We can easily extend our model to include nonstationary factors, by, for instance, choosing one or more of the $Y$ s to be drifted Brownian motions rather than Ornstein-Uhlenbeck processes. We will not discuss these modeling issues further here, but leave the analysis of this to the interested reader.

In the special case of a multivariate stochastic volatility Schwartz model (i.e. $m=0$ ) the 'reversion-adjusted' logreturns are approximately distributed according to a multivariate meanvariance mixture model. Considering the 'reversion-adjusted' logreturns over the time interval 
$[t, t+\tau]$, we find that

$$
\begin{aligned}
\ln \widetilde{S}(t+\tau)-\mathrm{e}^{A \tau} \ln \tilde{S}(t) & =X(t+\tau)-\mathrm{e}^{A \tau} X(t) \\
& =\int_{t}^{t+\tau} \mathrm{e}^{A(t+\tau-s)} \Sigma^{1 / 2}(s) \mathrm{d} W(s) \\
& \approx \mathrm{e}^{A \tau} \Sigma^{1 / 2}(t) \Delta_{\tau} W(t) .
\end{aligned}
$$

Here, $\Delta_{\tau} W(t):=W(t+\tau)-W(t)$ and $\tau$ is supposed to be sufficiently small in order to make the approximation above reasonable. Hence, we have 'reversion-adjusted' logreturns that are approximately distributed according to the multivariate mean-variance mixture model

$$
\left.\mathrm{e}^{A \tau} \Sigma^{1 / 2}(t) \Delta_{\tau} W(t)\right|_{\Sigma(t)} \sim \mathcal{N}\left(0, \mathrm{e}^{A \tau} \Sigma(t) \mathrm{e}^{A^{\top} \tau}\right)
$$

In [8] this was discussed in the univariate case, showing that we can choose stochastic volatility models yielding, for instance, normal inverse Gaussian distributed 'reversion-adjusted' returns. We refer the reader to [9] for a study of gas and oil prices where the normal inverse Gaussian distribution has been applied to model 'reversion-adjusted' returns. We further note that the conditional Gaussian structure of the 'reversion-adjusted' returns implies that the covariance determines the cross-commodity dependency. In this case it is given explicitly by the stochastic volatility model $\Sigma(t)$, introducing a time dependency in the covariance between commodities. In addition, the common factors $Y_{i}(t), i=1, \ldots, m$, will give codependent paths determined by common jump paths. Hence, we can mix rather complex dependency into the modeling. The autocovariance function of the deseasonalized logarithmic spot (14) gives explicit formulation to this dependence in terms of a second-order structure. For $h=0$, the autocovariance of deseasonalized logarithmic spots gives the covariance matrix of the deseasonalized logarithmic spots.

Let us discuss possible specifications of our spot price model satisfying the fundamental conditions on the operators and matrices in question. First of all, it is easily seen that if both of the matrices $A$ and $C_{j}$ are diagonal, then they will commute. In fact, supposing that $A$ is a diagonal matrix could be natural in view of interpreting the speed of mean reversion of each commodity modeled separately (as the corresponding entry on the diagonal), and not imposing any functional cross dependencies between the commodities. In such a model, dependencies will enter via the spike terms and in the stochastic volatility. When $A$ is diagonal, then all the diagonal elements must be negative in order to have negative eigenvalues (eigenvalues are equal to the diagonal elements in this case, of course). It is simple to see that the determinant of $A \otimes I+I \otimes A$ becomes

$$
\operatorname{det}(A \otimes I+I \otimes A)=2^{d} \prod_{i=1}^{d} a_{i} \prod_{i \neq j}^{d}\left(a_{i}+a_{j}\right)^{2},
$$

which is different from 0 since all the diagonal elements are strictly less than 0 . This means that $\boldsymbol{A}$ is invertible. In fact, when $C_{j}$ is also diagonal, we find that $\boldsymbol{A}-\boldsymbol{C}_{j}$ is invertible if and only if $a_{i}+a_{j} \neq c_{i}+c_{j}$ for $i, j=1, \ldots, d$. Note also that stationarity of the volatility holds only if all the diagonals of $C_{j}$ are strictly negative. But, this also implies that $\boldsymbol{C}_{j}$ is invertible.

When $A$ and $C_{j}$ are not diagonal matrices, rather strict conditions are imposed on the range of possible parameter choices to fulfill the commutativity condition. To investigate this in more detail, let us focus on $d=2$, that is, when $A$ and $C_{j}$ are elements in $M_{2}(\mathbb{R})$. Denoting the 
coordinates of $A$ and $C_{j}$ by $\left\{a_{k l}\right\}_{k, l=1,2}$ and $\left\{c_{k l}^{j}\right\}_{k, l=1,2}$, respectively, we find from matrix multiplication that $A C_{j}=C_{j} A$ if and only if

$$
\begin{aligned}
a_{12} c_{21}^{j} & =a_{21} c_{12}^{j}, \\
\left(a_{11}-a_{22}\right) c_{12}^{j} & =\left(c_{11}^{j}-c_{22}^{j}\right) a_{12}, \\
\left(a_{11}-a_{22}\right) c_{21}^{j} & =\left(c_{11}^{j}-c_{22}^{j}\right) a_{21} .
\end{aligned}
$$

For example, if $a_{12}=0$ then the first equation immediately yields either $c_{12}^{j}=0$ or $a_{21}=0$, or both. If $c_{12}^{j} \neq 0$ then the second equation gives $a_{11}=a_{22}$. In other words, in this particular situation the speed of mean reversion of each commodity must be the same. This is a severe modeling restriction. Of course, if $C_{j}$ is diagonal, we do not have such a restriction on the equality of the diagonal elements. In the situation where neither of the two matrices $A$ and $C_{j}$ are diagonal, the ratios $a_{12} / c_{12}^{j}$ and $a_{21} / c_{21}^{j}$ must be equal. Moreover, the ratio $\left(a_{11}-a_{22}\right) /\left(c_{11}^{j}-c_{22}^{j}\right)$ must be equal to $a_{12} / c_{12}^{j}$. These conditions must be satisfied in an estimation of the full model. Note that the determinant of $A \otimes I+I \otimes A$ becomes

$$
\operatorname{det}(A \otimes I+I \otimes A)=4 \operatorname{tr}(A)^{2} \operatorname{det}(A),
$$

and, hence, $\boldsymbol{A}$ is invertible if and only if $\operatorname{det}(A) \neq 0$ and $a_{11} \neq-a_{22}$. But, $A$ is supposed to be invertible, so $\operatorname{det}(A) \neq 0$. Furthermore, as the eigenvalues of $A$ are

$$
\lambda_{1,2}=\frac{1}{2} \operatorname{tr}(A) \pm \frac{1}{2} \sqrt{\operatorname{tr}(A)^{2}-4 \operatorname{det}(A)},
$$

we see that the real parts are negative if and only if $\operatorname{tr}(A)=a_{11}+a_{22}<0$. As long as we consider stationary models, $A$ is assumed to have eigenvalues with real part being less than 0 . Hence, in this case we find that $\boldsymbol{A}$ is invertible without any further conditions. The same holds for $\boldsymbol{C}_{j}$. However, we must have $\operatorname{tr}(A) \neq \operatorname{tr}\left(C_{j}\right)$ and $\operatorname{det}\left(A-C_{j}\right)$ in order for $\boldsymbol{A}-\boldsymbol{C}_{j}$ to be invertible. This puts additional conditions on $A$ and $C_{j}$. We note that going to the case $d=3$ significantly complicates the conditions of invertibility. We do not consider this or higher-order cases here.

Note that the matrices $B_{i}$ do not need to commute with $A$ and $C_{j}$. Hence, we do not have to place severe restrictions on the mixing of dependencies in the mean-reverting part of the $Y_{i}$ dynamics. If, for example, we want to model spikes using one or more factors $Y_{i}$, we can easily incorporate a direct functional dependency between spikes in different markets. For example, we may model co-jump behavior by letting power spikes also arise as a result of a spike in gas, and vice versa. In a model where we assume that the matrices $A$ and $C_{j}$ are diagonal (so that they commute), we can think of $X(t)$ as governing the price variations in 'nonspike' periods, where a dependency arises probabilistically via the stochastic volatility being driven by matrix-valued subordinators and the mixing of the Brownian motions in the dynamics of $X(t)$. When spikes occur, we may model a direct effect on the marginal spot prices. If, for instance, the gas prices spike, this will lead to spikes in electricity. This causal effect can be modeled by off-diagonal elements being nonzero in the matrix $B_{i}$.

\section{Simulation of matrix-valued subordinators}

In this section we discuss simulation of our spot price dynamics, which essentially means discussing the simulation of matrix-valued subordinators.

Limited literature is available on the simulation of matrix-valued subordinators. One possible approach could be to apply existing methods to sample multivariate Lévy processes based on 
their Lévy measures by iterative sampling from the conditional marginals (see, e.g. [16]). However, the marginal distribution functions are required, which are not always available in a simple form. Moreover, in case of matrix-valued subordinators, the restriction of the domain to the positive definite cone makes matters even more complicated. We introduce a simple approximative algorithm, kindly proposed to us by Robert Stelzer, to simulate from matrix-valued compound Poisson, stable, and tempered stable processes with stable or constant jump size distribution.

For any $U \in \mathbb{S}_{d}^{+}$, we can make a polar decomposition in a ray $r=\|U\|=\operatorname{tr}\left(U^{\top} U\right)^{1 / 2}$ and angle $\Theta=U / r$, so that $U=r \Theta$. Moreover, $\Theta$ is situated on the unit sphere $S$ of $\mathbb{R}^{d \times d}$ intersected with the positive definite cone, i.e. $\Theta \in S \mathbb{S}_{d}^{+}:=\operatorname{vec}^{-1} \boldsymbol{S} \cap \mathbb{S}_{d}^{+}$. into

Suppose that $v$ is a Lévy measure on $\mathbb{S}_{d}^{+}$of the subordinator $L$, such that it can be decomposed

$$
v(\mathrm{~d} U)=\Gamma(\mathrm{d} \Theta) \widetilde{v}(\Theta, \mathrm{d} r), \quad U \in \mathbb{S}_{d}^{+},
$$

where $\widetilde{v}(\Theta, \mathrm{d} r)$ is a Lévy measure on $\mathbb{R}_{+}$and $\Gamma$ is a spectral measure on $\boldsymbol{S S}_{d}^{+}$concentrated on a finite number of points $\left\{\Theta_{i}\right\}_{1 \leq i \leq p}$. Note in passing that any measure can be approximated by a measure concentrated on finitely many points. Since $L$ is a pure jump subordinator, its cumulant function is given by

$$
\begin{aligned}
\phi_{L(1)}(V) & =\int_{\mathbb{S}_{d}^{+} \backslash\{0\}}\left(\mathrm{e}^{\mathrm{i} \operatorname{tr}(V U)}-1\right) v(\mathrm{~d} U) \\
& =\int_{S \mathbb{S}_{d}^{+}} \int_{0}^{\infty}\left(\mathrm{e}^{\mathrm{i} r \operatorname{tr}(V \Theta)}-1\right) \widetilde{\mathcal{v}}(\Theta, \mathrm{d} r) \Gamma(\mathrm{d} \Theta) \\
& =\sum_{i=1}^{p} \Gamma\left(\Theta_{i}\right) \int_{0}^{\infty}\left(\mathrm{e}^{\mathrm{i} r \operatorname{tr}\left(V \Theta_{i}\right)}-1\right) \widetilde{v}\left(\Theta_{i}, \mathrm{~d} r\right) .
\end{aligned}
$$

One recognizes this as the cumulant of a weighted sum of $p$ independent, real-valued subordinator processes. This leads to the following simple algorithm to sample $L$ according to its cumulant function.

- Find the finite set of points $\{\Theta\}_{1 \leq i \leq n}$ where $\Gamma$ is concentrated.

- Simulate $p$ independent subordinators $R_{i}(t)$ with cumulant function

$$
\phi_{R_{i}(1)}\left(\operatorname{tr}\left(V \Theta_{i}\right)\right)=\int_{0}^{\infty}\left(\mathrm{e}^{\mathrm{i} r \operatorname{tr}\left(V \Theta_{i}\right)}-1\right) \widetilde{v}\left(\Theta_{i}, \mathrm{~d} r\right) .
$$

- Set $L(t)=\sum_{i=1}^{p} R_{i}(t) \Theta_{i}$.

To make this algorithm operationable, we must be able to sample the $R_{i} \mathrm{~s}$, which we now discuss in particular cases which are of interest in energy markets.

First, let us consider a matrix-valued compound process (mCP) with only positive jumps $L$. This becomes a multivariate compound Poisson process restricted to values in the symmetric positive definite cone. Its cumulant function is

$$
\phi_{L(1)}(V)=\lambda \int_{\mathbb{S}_{d}^{+}}\left(\mathrm{e}^{\mathrm{itr}(V U)}-1\right) v(\mathrm{~d} U),
$$

where $v$ is the jump size distribution and $\lambda$ is the intensity. Supposing that $v(\mathrm{~d} U)=\widetilde{v}(\Theta, \mathrm{d} r)$ $\Gamma(\Theta)$ with $\widetilde{v}(\Theta, \mathrm{d} r)$ being a probability distribution on $\mathbb{R}_{+}$and $\Gamma(\mathrm{d} \Theta)$ for a spectral measure 
$\Gamma$ on $S_{\mathbb{S}_{d}^{+}}^{+}$, concentrated on finitely many points, it holds that

$$
\phi_{L(1)}(V)=\lambda \sum_{i=1}^{p} \Gamma\left(\Theta_{i}\right) \int_{0}^{\infty}\left(\mathrm{e}^{\mathrm{i} r \operatorname{tr}\left(V \Theta_{i}\right)}-1\right) \widetilde{v}\left(\Theta_{i}, \mathrm{~d} r\right)
$$

Hence, $R_{i}$ for $i=1, \ldots, p$ will follow a one-dimensional compound Poisson process with jump intensity $\lambda \Gamma\left(\Theta_{i}\right)$ and jump size distribution $\widetilde{v}\left(\Theta_{i}, \mathrm{~d} r\right)$. The $\mathrm{mCP}(\lambda)$ process $L$ is represented as a linear combination of angles $\Theta_{i}$ and radius processes being one-dimensional compound Poisson processes $R_{i}$, i.e. $L(t)=\sum_{i=1}^{p} R_{i}(t) \Theta_{i}$.

By exponential tilting of matrix-valued $\alpha$-stable laws, a multivariate extension of tempered stable laws can be made. The inverse Gaussian distribution is a special case of this class of functions. The polar decomposition of the Lévy measure $v$ of a matrix-valued tempered $\alpha / 2$-stable law is given by (see [1])

$$
v(\mathrm{~d} U)=\frac{\mathrm{e}^{-r \operatorname{tr}(\Delta \Theta)}}{r^{1+\alpha / 2}} \mathrm{~d} r \Gamma(\mathrm{d} \Theta) .
$$

In the case $\alpha=1, v$ is a Lévy measure of a matrix extension of the inverse Gaussian distribution (mIG), where $\Delta \in \mathbb{S}_{d}^{+}$and $\Gamma$, a finite measure on $S_{\mathbb{S}_{d}^{+}}^{+}$, are parameters. As in the univariate case, the inverse Gaussian process is a pure jump process; hence, the cumulant function is given by

$$
\phi_{L(1)}(V)=\int_{S S_{d}^{+}} \int_{0}^{\infty}\left(\mathrm{e}^{\mathrm{i} r \operatorname{tr}(V \Theta)}-1\right) \mathrm{e}^{-r \operatorname{tr}(\Delta \Theta)} \frac{\mathrm{d} r}{r^{3 / 2}} \Gamma(\mathrm{d} \Theta)+\mathrm{i} \operatorname{tr}\left(V \mu_{0}\right)
$$

for $L(1) \sim \operatorname{mIG}\left(\Delta, \Gamma, \mu_{0}\right)$, where $\mu_{0} \in \mathbb{S}_{d}^{+}$is a parameter. Choosing $\Gamma$ such that it is concentrated on finitely many points and decomposing $\mu_{0}$ in an angle $\Theta_{0} \in S \mathbb{S}_{d}^{+}$and a radius $r_{0} \in \mathbb{R}$ leads to

$$
\phi_{L(1)}(V)=\sum_{i=1}^{p} \Gamma\left(\Theta_{i}\right) \int_{0}^{\infty}\left(\mathrm{e}^{\mathrm{i} r \operatorname{tr}\left(V \Theta_{i}\right)}-1\right) \mathrm{e}^{-r \operatorname{tr}\left(\Delta \Theta_{i}\right)} \frac{\mathrm{d} r}{r^{3 / 2}}+\mathrm{i} r_{0} \operatorname{tr}\left(\Phi \Theta_{0}\right) .
$$

One can compare this with the characteristic function of a one-dimensional inverse Gaussian random variable $G$, for which the cumulant function is given by

$$
\phi_{G}(\zeta)=\mathrm{i} \frac{\delta}{\gamma}(2 \mathcal{N}(\gamma)-1) \zeta+\frac{\delta}{\sqrt{2 \pi}} \int_{0}^{\infty}\left(\mathrm{e}^{\mathrm{i} \zeta x}-1\right) \mathrm{e}^{-1 / 2 \gamma^{2} x} \frac{\mathrm{d} x}{x^{3 / 2}}, \quad \zeta \in \mathbb{R},
$$

where $\mathcal{N}$ denotes the cumulative normal distribution. We recognize $L$ as a matrix of linear combinations of a finite number of angles $\Theta_{i}, i=1, \ldots, p$, with coefficients given by one-dimensional inverse Gaussian subordinator processes $R_{i}(t)$, where $R_{i}(1)$ is distributed according to the inverse Gaussian distribution $\operatorname{IG}\left(\delta_{i}, \gamma_{i}\right)$, where $\delta_{i}=\sqrt{2 \pi} \Gamma\left(\Theta_{i}\right)$ and $\gamma_{i}=$ $2 \sqrt{\operatorname{tr}\left(\Delta \Theta_{i}\right)}$. Moreover, the drift parameter $\mu_{0}$ of the multivariate inverse Gaussian distribution is by default chosen such that the drift term of the $\mathrm{mIG}$ distribution equals the drift term of $\sum_{i=1}^{p} R_{i}(t) \Theta_{i}$.

As an example, consider the case of two spot prices $S_{1}(t)$ and $S_{2}(t)$ modeled by our dynamics. We have in mind the spot price of electricity in two interconnected markets, or the spot price of gas and electricity. We suppose that the prices are driven by two $M_{2}(\mathbb{R})$-valued subordinator processes $\widetilde{L}_{1}(t)$ and $\widetilde{L}_{2}(t)$. The first process defines the spike component, while the second part determines the stochastic volatility. We assume that there is one spike component $Y(t) \in \mathbb{R}^{2}$, 
while the stochastic volatility process $\Sigma(t)$ is an equally weighted sum of two processes $Z_{1}(t)$ and $Z_{2}(t)$, whose dynamics are driven by $\widetilde{L}_{1}$ and $L_{2}$, respectively. The dynamics of the spike process $Y(t)$ are driven by the diagonal of $\widetilde{L}_{1}(t)$. In order to make simulations from the model, we must specify the parameters. To simplify, we set the seasonality function identically equal to 1 , that is, $\Lambda_{i}(t)=1$ for $i=1,2$. Moreover, we choose

$$
\begin{gathered}
A=\left(\begin{array}{ll}
-1.4 & -0.3 \\
-0.3 & -1.4
\end{array}\right), \quad B=\left(\begin{array}{cc}
-2 & 1 \\
1 & -2
\end{array}\right), \quad \eta=\left(\begin{array}{cc}
1 & 0.5 \\
0.5 & 1
\end{array}\right), \\
C_{1}=\left(\begin{array}{cc}
-0.4 & 0.3 \\
0.3 & -0.4
\end{array}\right), \quad C_{2}=\left(\begin{array}{cc}
-0.045 & 0.03 \\
0.03 & -0.045
\end{array}\right) .
\end{gathered}
$$

We let the levels of the spike component $Y(t)$ be $0, \mu_{1}=\mu_{2}=0$.

We see that the $A$ matrix has rather high values on the diagonal, meaning that logarithmic deseasonalized prices are reverting fast. Such a feature has been observed in several empirical studies of electricity spot prices quoted on the German power market EEX (see, for example, [12]). By letting the off-diagonal coordinates be nonzero, we incorporate a functional dependency between the two prices. Here it is assumed that $X_{1}$ is negatively reacting to changes in $X_{2}$ and vice versa. Similarly, the spike component $Y$ is mean reverting quickly, naturally at a faster speed than the logarithmic prices themselves. This is in accordance with how one observes the spike behavior in electricity prices (see [12] again). If one thinks of gas being a fuel for electricity generation, it is natural to have spikes occurring in electricity prices whenever they happen in gas. Moreover, as demand for electricity increases with cold weather, it also naturally impacts the demand for gas. Here we mimic such spike dependency by letting the $B$ matrix have positive values on the off-diagonal. Moreover, we have also supposed a statistical dependency in the spike processes by letting the $\eta$ matrix have off-diagonals being nonzero (and positive). The matrices $C_{1}$ and $C_{2}$ for the stochastic volatility process $\Sigma$ have considerably slower speeds of mean reversion. The parameters for the stochastic volatility part were inspired by the empirical study in [33]. Vos [33] fitted a two-factor BNS stochastic volatility model to Dutch stock price data. We have applied these numbers here simply for illustration, and do not intend to claim that they are necessarily relevant for energy markets.

Note that all the defined matrices are invertible and have two negative real eigenvalues. Moreover, for $j=1,2, A$ and $C_{j}$ commute. Finally, as $\operatorname{det}\left(A-C_{j}\right)>0$, we find that $\boldsymbol{A}-\boldsymbol{C}_{j}$ is invertible. Hence, our specified model satisfies the main assumptions in this paper.

Next, let us define the two subordinator processes $\widetilde{L}_{1}(t)$ and $\widetilde{L}_{2}(t)$. To mimic spikes in the market, we consider a simple Poisson process for $\widetilde{L}_{1}(t)$. To have a stochastic volatility process which can generate adjusted returns being close to NIG distributed, we suppose that $\widetilde{L}_{2}(t)$ is mIG. The NIG distribution has been shown to fit electricity prices in several empirical studies (see [5], [9], [10], and [14]). In order to be able to simulate these two processes, we apply the idea above, and define a simple discrete spectral measure on $\boldsymbol{S} \mathbb{S}_{2}^{+}$. It is easy to see that

$$
\Theta=\left(\begin{array}{cc}
\theta & \pm \sqrt{\theta(1-\theta)} \\
\pm \sqrt{\theta(1-\theta)} & 1-\theta
\end{array}\right) \quad \text { or } \quad\left(\begin{array}{cc}
\theta & 0 \\
0 & \sqrt{1-\theta^{2}}
\end{array}\right)
$$

for $\theta \in(0,1)$ are three valid choices of $\Theta \in S \mathbb{S}_{2}^{+}$. To this end, we discretize the unit interval with step size 0.1 , and choose $\theta_{j}=j \times 0.1$ for $j=1, \ldots, 9$. We choose either one of the three possible matrix structures for $\Theta$ with given $\theta_{i}$, making up a total of 27 matrices $\Theta_{i}$. For the Poisson process, we choose the intensity such that $\lambda \Gamma\left(\Theta_{i}\right)=\frac{3}{100}$ and the jump size distribution fixed to be 1.7, that is, if $R_{i}(t)$ is jumping at time $t$, then $\Delta R_{i}(t)=1.7$. This will correspond to 


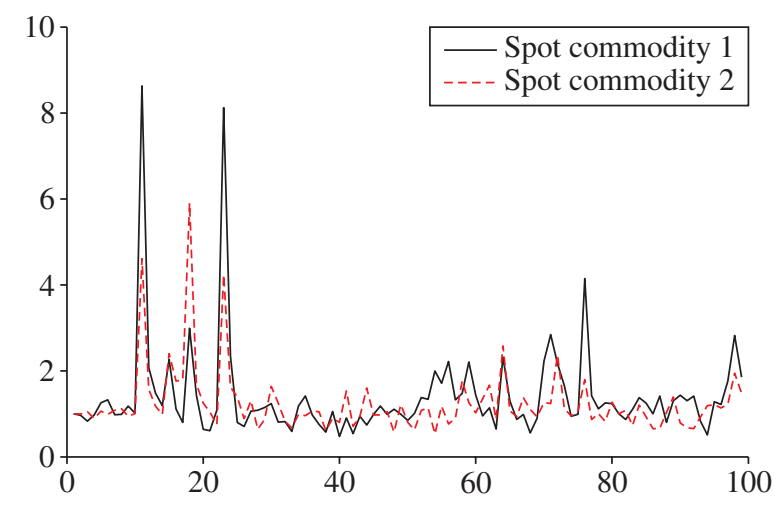

Figure 1: Simulated spot prices of the two commodities.

a change in the spot price by a factor $\exp (1.7)=5.47$, which is a rather dramatic price change. As a measure for the mIG part, we set $\Gamma\left(\Theta_{i}\right)=1 / 324 \sqrt{2 \pi}$ uniformly for all $1 \leq i \leq 27$. Finally, we suppose that the parameter $\Delta$ of the $\mathrm{mIG}$ part is

$$
\Delta=\left(\begin{array}{ll}
50 & 45 \\
45 & 50
\end{array}\right) .
$$

In Figure 1 the spot price series resulting from our two-dimensional example is shown, where we have used an Euler scheme to discretize the dynamics in time and standard schemes for the sampling of an inverse Gaussian distributions (see [27]). One can clearly see the dependency between the two spot prices, in particular, how the spikes follow each other in the two series. The high speed of mean reversion in the $Y$ process is recognized in the rapid decay of the spikes. We also observe that the price series possesses idiosyncratic risk and large variations in 'nonspike' periods.

\section{Conclusions}

We have proposed a model to describe the spot price dynamics for cross-commodity markets in a multivariate setting. The model captures features such as mean reversion, spikes, stochastic volatility, and inverse leverage effect. The dynamics are a multidimensional extension of the Barndorff-Nielsen and Shephard stochastic volatility model embedded into mean-reversion dynamics relevant for commodity price series. The choice of the multidimensional extension is influenced by the work of Stelzer [31]. The multivariate spot model is analytically tractable and probabilistic properties can to a large degree be explicitly computed. We have derived various characteristics, such as stationary distributions and covariance functions. The model is a multivariate extension of the one-dimensional spot price dynamics analyzed by Benth [8].

A simple approximative algorithm to simulate from matrix-valued subordinators was introduced. The method was demonstrated on an empirical example. However, further research has to be done to generate matrix-valued Lévy processes in a more general setting; a study we leave for the future.

As far as we know, there exists no methods to estimate the model based on spot price data. It is obviously of crucial interest for the applicability of the model to understand how to fit the parameters to data. Methods are available to estimate the model in the diffusion case on the quadratic covariation [3]. However, these methods require high frequency data, which does 
not exist in the energy market. Another alternative is to adopt the methods already available for filtering spike data from price series into a multidimensional setting. If this is possible then the estimation of the spike process can be treated separately from the diffusion part, and the diffusion part can be estimated conditionally on the spike parameters. Before this can be implemented, further research has to be done on the validity of these methods. Another possibility is to estimate directly on the characteristic function in the frequency domain.

\section{References}

[1] Barndorff-Nielsen, O. E. And Pérez-Abreu, V. (2002). Extension of type $G$ and marginal infinite divisibility. Theory Prob. Appl. 47, 202-218.

[2] Barndorff-Nielsen, O. E. And Pérez-Abreu, V. (2008). Matrix subordinators and related upsilon transformations. Theory Prob. Appl. 52, 1-23.

[3] Barndorff-Nielsen, O. E. AND Shephard, N. (2001). Econometric analysis of realized covariation: high frequency based covariance, regression, and correlation in financial economics. Econometrica 72, 885-925.

[4] BarndorfF-Nielsen, O. E. AND Shephard, N. (2001). Non-Gaussian Ornstein-Uhlenbeck-based models and some of their uses in economics. J. R. Statist. Soc. B 63, 167-241.

[5] Barndorff-Nielsen, O. E., Benth, F. E. and Veraat, A. E. D. (2013). Modelling energy spot prices by Lévy semistationary processes. To appear in Bernoulli.

[6] Barndorff-Nielsen, O. E., Pedersen, J. and Sato, K.-I. (2001). Multivariate subordination, self-decomposability and stability. Adv. Appl. Prob. 33, 160-187.

[7] Barndorff-Nielsen, O. E. And Stelzer, R. (2007). Positive-definite matrix processes of finite variation. Prob. Math. Statist. 27, 3-43.

[8] BENTH, F. E. (2011). The stochastic volatility model of Barndorff-Nielsen and Shephard in commodity markets. Math. Finance 21, 595-625.

[9] Benth, F. E. And ŠAltytĖ-Benth, J. (2004). The normal inverse Gaussian distribution and spot price modeling in energy markets. Internat. J. Theoret. Appl. Finance 7, 177-192.

[10] Benth, F. E. And Schmeck, M. D. (2012). Pricing futures and options in electricity markets. Submitted.

[11] Benth, F. E. ANd Vos, L. (2013). Pricing of forwards and options in a multivariate non-Gaussian stochastic volatility model for energy markets. Adv. Appl. Prob. 45, 572-594.

[12] Benth, F. E., Kiesel, R. and Nazarova, A. (2012). A critical empirical study of three electricity spot price models. Energy Econom. 34, 1589-1616.

[13] Benth, F. E., Š Altytė Benth, J. And Koekebakker, S. (2008). Stochastic Modelling of Electricity and Related Markets. World Scientific, Hackensack, NJ.

[14] Börger, R., Cartea, A., Kiesel, R., and Schindlmayer, G. (2009). Cross-commodity analysis and applications to risk management. J. Futures Markets 29, 197-217.

[15] Burger, M., Klar, B., Müller, A. And Schindlmayer, G. (2004). A spot market model for pricing derivatives in electricity markets. Quant. Finance 4, 109-122.

[16] Cont, R. And Tankov, P. (2004). Financial Modelling with Jump Processes. Chapman \& Hall/CRC, Boca Raton, FL.

[17] Deaton, A. and Leroque, G. (1992). On the behavior of commodity prices. Rev. Econom. Studies 59, 1-23.

[18] Eydeland, A. And Wolyniec, K. (2003). Energy Power and Risk Management. John Wiley, Chichester.

[19] Geman, H. (2005). Commodities and Commodity Derivatives. John Wiley, Chichester.

[20] Hikspoors, S. and Jaimungal, S. (2008). Asymptotic pricing of commodity derivatives using stochastic volatility spot models. Appl. Math. Finance 15, 449-477.

[21] IKeda, N. And Watanabe, S. (1981). Stochastic Differential Equations and Diffusion Processes. North-Holland, Amsterdam.

[22] Jacod, J. And Shiryaev, A. N. (1987). Limit Theorems for Stochastic Processes. Springer, Berlin.

[23] Muhle-Karbe, J., Pfaffel, O. and Stelzer, R. (2012). Option pricing in multivariate stochastic volatility models of OU type. SIAM J. Financial Math. 3, 66-94.

[24] Pigorsch, C. and Stelzer, R. (2009). A multivariate Ornstein-Uhlenbeck type stochastic volatility model. Eprint. Available at http://www-m4.ma.tum.de.

[25] Pigorsch, C. And Stelzer, R. (2009). On the definition, stationary distribution and second order structure of positive semidefinite Ornstein-Uhlenbeck type processes. Bernoulli 15, 754-773.

[26] Protter, P. (1990). Stochastic Integration and Differential Equations. Springer, Berlin.

[27] Rydberg, T. H. (1997). The normal inverse Gaussian Lévy process: simulation and approximation. Commun. Statist. Stoch. Models 13, 887-910.

[28] SATo, K.-I. (2004). Stochastic integrals in additive processes and application to semi-Lévy processes. Osaka J. Math. 41, 211-236. 
[29] Schwartz, E. S. (1997). The stochastic behavior of commodity prices: implications for valuation and hedging. J. Finance 52, 923-973.

[30] Singleton, K. J. (2001). Estimation of affine asset pricing models using the empirical characteristic function. J. Econometrics 102, 111-141.

[31] Stelzer, R. J. (2007). Multivariate continuous time stochastic volatility models driven by a Lévy process. Doctoral Thesis, TU Munchen. Available at http://mediatum2.ub.tum.de/doc/624065/.

[32] Trolle, A. B. and Schwartz, E. S. (2009). Unspanned stochastic volatility and the pricing of commodity derivatives. Rev. Financial Studies 22, 4423-4461.

[33] Vos, L. (2009). Path-dependent options and the effect of stochastic volatility. Unpublished manuscript. 